\title{
RELATIVE ALGEBRO-GEOMETRIC STABILITIES OF TORIC MANIFOLDS
}

\author{
NAOTO YOTSUTANI AND BIN ZHOU
}

\begin{abstract}
In this paper we study the relative Chow and $K$-stability of toric manifolds in the toric sense. First, we give a criterion for relative $K$-stability and instability of toric Fano manifolds in the toric sense. The reduction of relative Chow stability on toric manifolds will be investigated using the Hibert-Mumford criterion in two ways. One is to consider the maximal torus action and its weight polytope. We obtain a reduction by the strategy of Ono [33], which fits into the relative GIT stability detected by Székelyhidi. The other way relies on $\mathbb{C}^{*}$-actions and Chow weights associated to toric degenerations following Donaldson and Ross-Thomas [12, 35]. In the end, we determine the relative $K$-stability of all toric Fano threefolds and present counter-examples which are relatively $K$-stable in the toric sense but which are asymptotically relatively Chow unstable.
\end{abstract}

\section{INTRODUCTION}

The well-known Yau-Tian-Donaldson conjecture asserts that a compact complex polarized manifold $(X, L)$ admits canonical metrics (Kähler-Einstein metrics, constant scalar curvature (cscK) metrics, and extremal metrics, etc) in $2 \pi c_{1}(L)$ if and only if $(X, L)$ is stable in the sense of Geometric Invariant Theory. Among various notions of stability, $K$-stability and Chow stability are the most widely studied. Many authors use the term polystability rather than stability, since the former agrees better with the notions in GIT. Throughout this paper, we use the latter for simplicity.

The conception of $K$-stability was first introduced by Tian [40] in the study of the existence of Kähler-Einstein metrics in the first Chern class (if it is positive) on a Kähler manifold. Later, Donaldson extended it to general polarized varieties [12] and made a conjecture on the relation between $K$-stability and the existence of cscK metrics. More generally, for the existence of extremal metrics, the definition of $K$-stability was extended by Székelyhidi [39] to Kähler classes with non-vanishing Futaki invariant and was called relative $K$-stability. Meanwhile, the conception of Chow stability is also significant in Kähler geometry. Let $\operatorname{Aut}(X, L)$ be the automorphism group of $(X, L)$. In [11] Donaldson showed that the existence of a cscK metric in $2 \pi c_{1}(L)$ implies the asymptotic Chow stability of $(X, L)$ if $\operatorname{Aut}(X, L)$ is discrete. Donaldson's result was generalized by Mabuchi [24], with the assumption on $\operatorname{Aut}(X, L)$ replaced by the condition of vanishing higher order Futaki invariants. Very recently, it has been shown that the existence of extremal metrics implies asymptotically Chow stability relative to a maximal torus [26, 36]. With these remarkable progress, the verification of the stabilities is drawing more and more attention. In general, this is a complicated problem since one has to study an infinite number

Date: December 20, 2016.

Key words and phrases. Extremal metrics, $K$-stability, Chow stability, toric manifold.

The second author was supported partially by ARC grant DE120101167, NSFC grants No. 11571018 and 11331001 . 
of possible degenerations of the manifold. In this paper, we shall discuss the stabilities of toric manifolds.

For toric manifolds, a well-understood reduced version of the relative $K$-stability on the moment polytope is believed to be equivalent to the existence of extremal metrics [12, 47]. This conjecture has been confirmed for toric surfaces [13, 14, 6].

Let $\left(X_{\Delta}, L_{\Delta}\right)$ be the polarized toric manifold which corresponds to a polytope $\Delta$

$$
\left\langle l_{i}, x\right\rangle \leq \lambda_{i}, i=1, \ldots, d,
$$

satisfying Delzant's condition, where $\lambda_{i} \in \mathbb{Z}, l_{i} \in \mathbb{Z}^{n}$ is primitive. Without loss of generality, we assume 0 lies in the interior of $\Delta$. Let $\theta_{\Delta}$ be the potential function of the extremal vector field $V$ [17], which is affine linear on $\Delta$, and normalized by $\int_{\Delta} \theta_{\Delta} d x=0$ (see Lemma 2.1). In [12], Donaldson reduced $K$-stability to the positivity of a linear functional defined on $\Delta$. This functional was generalized for relative $K$-stability and is given by [47]

$$
\mathcal{L}_{\Delta}(u)=\int_{\partial \Delta} u d \sigma-\int_{\Delta}\left(\bar{S}+\theta_{\Delta}\right) u d x
$$

where $\bar{S}$ is the average of the scalar curvature, and $d \sigma=\left|l_{i}\right|^{-1} d \sigma_{0}$ on the face in $\left\{x \in \mathbb{R}^{n}\right.$ : $\left.\left\langle l_{i}, x\right\rangle=\lambda_{i}\right\}$. Here $d \sigma_{0}$ is the standard Lebesgue measure on $\partial \Delta$. Note that this functional corresponds to the modified Futaki invariant in [39].

Recall that a convex function $u$ is piecewise linear if there are affine linear functions $f_{1}, \ldots, f_{\ell}$ such that $u=\max \left\{f_{1}, \ldots, f_{\ell}\right\}$. Furthermore $u$ is simple piecewise linear if it is of the form $u=\max \{0, f\}$ for a linear function $f$. In view of [12, 39, 47, 48], we have:

Definition 1.1. A toric manifold $\left(X_{\Delta}, L_{\Delta}\right)$ is called relatively $K$-semistable in the toric sense if $\mathcal{L}_{\Delta}(u) \geqslant 0$ for all piecewise linear convex functions. Furthermore, it is called relatively $K$-stable in the toric sense when $\mathcal{L}_{\Delta}(u)=0$ if and only if $u$ is affine linear.

When proving the existence of cscK metrics on toric surfaces [13, 14] Donaldson introduced a stronger notion called strong $K$-stability. Let $\Delta^{*}$ be the union of the interior of $\Delta$ and the interiors of its co-dimension 1 faces. Denote

$$
\mathcal{C}_{1}=\left\{u \mid u \text { is convex on } \Delta^{*} \text { and } \int_{\partial \Delta} u<\infty\right\} .
$$

The linear functional $\mathcal{L}_{\Delta}$ is well-defined on $\mathcal{C}_{1}$.

Definition 1.2. $\left(X_{\Delta}, L_{\Delta}\right)$ is called relatively strongly $K$-stable in the toric sense if $\mathcal{L}_{\Delta}(u) \geqslant$ 0 for all convex functions in $\mathcal{C}_{1}$ and $\mathcal{L}_{\Delta}(u)=0$ if and only if $u$ is an affine linear function.

To check the positivity of $\mathcal{L}_{\Delta}$ in dimension 2 , it suffices to consider simple piecewise linear convex functions on $\Delta[12,44]$. In higher dimensions, a sufficient condition for the relative strong $K$-stability in the toric sense was given by [47]. When $X$ is a Fano $n$-fold, $L$ is the anti-canonical line bundle and $\bar{S}=n$ the sufficient condition is

$$
\sup _{\Delta} \theta_{\Delta} \leqslant 1
$$

Condition (1.3) has been verified for all toric Fano surfaces. In [47], it was also asked whether it holds for higher dimensions or not. Furthermore, Mabuchi proposed the following question when considering the existence of extremal metrics on toric Fano manifolds.

Problem 1.3. Let $\left(X_{\Delta}, L_{\Delta}\right)$ be a polarized toric Fano manifold. Is $\left(X_{\Delta}, L_{\Delta}\right)$ always relatively $K$-stable or not? 
If the answer were affirmative, one can expect that any toric Fano manifold admits an extremal metric like the case of Kähler-Ricci solitons [45]. However, we have found counterexamples (Corollary 1.6). By a simple observation and together with (1.3), we have:

Theorem 1.4. Assume $\left(X_{\Delta}, L_{\Delta}\right)$ is a toric Fano manifold and $\theta_{\Delta}=\sum_{i=1}^{n} a_{i} x_{i}+c$, where $a_{i}, c \in \mathbb{R}$. Let $\Delta^{-}=\left\{x \in \Delta: 1-\theta_{\Delta}<0\right\}$.

(1) If $\operatorname{Int}\left(\Delta^{-}\right)=\emptyset$, i.e. $\theta_{\Delta} \leq 1$ on $\Delta$, then $\left(X_{\Delta}, L_{\Delta}\right)$ is relatively strongly $K$-stable in the toric sense. Here $\operatorname{Int}\left(\Delta^{-}\right)$is the interior of $\Delta^{-}$.

(2) If $\operatorname{Int}\left(\Delta^{-}\right) \neq \emptyset$ and satisfies

$$
1-c<\frac{\int_{\Delta^{-}}\left(1-\theta_{\Delta}\right)^{2} d x}{\operatorname{Vol}\left(\Delta^{-}\right)}
$$

then there exists a simple piecewise linear function such that $\mathcal{L}_{\Delta}(u)<0$.

An application of Theorem 1.4 is to determine relative $K$-stablities of all toric Fano threefolds. The condition $\theta_{\Delta} \equiv 0$ is equivalent to the vanishing of the Futaki invariant [17]. Note that there are 18 toric Fano threefolds (see Section 2.1). Among all of them, $\mathbb{C} P^{3}, \mathcal{B}_{4}$, $\mathcal{C}_{3}, \mathcal{C}_{5}$ and $\mathcal{F}_{1}$ have a vanishing Futaki invariant, so condition $(1.3)$ is true. By computation with Theorem 1.4, we have:

Theorem 1.5. Let $X$ be a toric Fano threefold. We assume that the Futaki invariant of $X$ does not vanish. Then $X$ is relatively strongly $K$-stable in the toric sense in the anticanonical class if and only if $X$ is one of the following: $\mathcal{B}_{2}, \mathcal{B}_{3}, \mathcal{C}_{1}, \mathcal{C}_{4}, \mathcal{E}_{3}, \mathcal{E}_{4}$ and $\mathcal{F}_{2}$.

It is known that all toric Fano surfaces admit extremal metrics in the anti-canonical class [5, 7]. The instability tells us that counter-examples appear in dimension 3.

Corollary 1.6. If $X$ is one of $\mathcal{B}_{1}, \mathcal{C}_{2}, \mathcal{D}_{1}, \mathcal{D}_{2}, \mathcal{E}_{1}$ and $\mathcal{E}_{2}$, then $X$ does not admit extremal metrics in its first Chern class.

On the other hand, the reduction of Chow stability is also an interesting problem. A natural idea is to use the Hilbert-Mumford criterion. Ono [33] studied Chow stability of toric manifolds by considering the maximal torus action and its weight polytope. He obtained a reduction by adapting Gelfand-Kapranov-Zelevinsky's theory of Chow polytopes [19. 21]. He also defined a notion of the relative Chow semistability in the toric sense. In this paper, we introduce a refinement of this notion so that it fits naturally into the relative GIT stability detected by Székelyhidi [38, Chapter 1].

Let $\left(X_{\Delta}, L_{\Delta}\right)$ be a polarized toric manifold and $N=\operatorname{dim}\left(H^{0}\left(X_{\Delta}, L_{\Delta}\right)\right)-1$. We consider the relative Chow stability of $X_{\Delta} \subset \mathbb{C} P^{N}$ (see Section 3.1 for definitions). Now we assume $G=\left(\mathbb{C}^{*}\right)^{N+1}$ is a subgroup of diagonal matrices in $\mathrm{GL}(N+1, \mathbb{C})$. Following [33], we only consider the specific maximal torus of $\operatorname{SL}(N+1, \mathbb{C})$ which is also a subtorus of $\left(\mathbb{C}^{*}\right)^{N+1}$ given by

$$
T_{\Delta}^{\mathbb{C}}=\left\{\left(t_{1}, \ldots, t_{N+1}\right) \in\left(\mathbb{C}^{*}\right)^{N+1} \mid \prod_{j=1}^{N+1} t_{j}=1\right\} .
$$

Let $\beta$ be the $\mathbb{C}^{*}$-action induced by the extremal vector field $V$ as in Section 2.2

Definition 1.7. $\left(X_{\Delta}, L_{\Delta}\right)$ is relatively Chow semistable(stable, unstable) in the toric sense if the Chow form is $T_{\Delta}^{\mathbb{C}}$-semistable(stable, unstable) relative to $\beta$. 
Finally we consider the asymptotic relative Chow stability. Denote the Ehrhart polynomial of $\Delta$ by $E_{\Delta}(t)$. For any $i \in \mathbb{Z}_{+}$, we replace $\Delta$ above by $i \Delta, N+1$ by $E_{\Delta}(i)$ and $G=$ $\left(\mathbb{C}^{*}\right)^{E_{\Delta}(i)}$. Then we consider the maximal diagonalized torus $T_{i \Delta}^{\mathbb{C}}:=G \cap \operatorname{SL}\left(E_{\Delta}(i), \mathbb{C}\right)$.

Definition 1.8. $\left(X_{\Delta}, L_{\Delta}\right)$ is asymptotically relatively Chow semistable (stable, unstable) in the toric sense if the Chow form is $T_{i \Delta}^{\mathbb{C}}$-semistable (stable, unstable) relative to $\beta$ for all sufficiently large $i$.

In this paper, we will describe the asymptotic relative Chow stability in the toric sense in a combinatorial way. The character group $\chi(G)$ of $G$ is identified with

$$
\left\{i \Delta \cap \mathbb{Z}^{n} \rightarrow \mathbb{Z}\right\} \cong\left\{\Delta \cap(\mathbb{Z} / i)^{n} \rightarrow \mathbb{Z}\right\} \cong \mathbb{Z}^{E_{\Delta}(i)}
$$

For future convenience, we denote $\chi(G) \otimes \mathbb{R}$ by

$$
W(i \Delta):=\left\{i \Delta \cap \mathbb{Z}^{n} \rightarrow \mathbb{R}\right\} \cong\left\{\Delta \cap(\mathbb{Z} / i)^{n} \rightarrow \mathbb{R}\right\} \cong \mathbb{R}^{E_{\Delta}(i)} .
$$

As in [19, p.220], we identify $W(i \Delta)$ with its dual space by the scalar product

$$
\langle\varphi, \psi\rangle=\sum_{\mathbf{a} \in \Delta \cap(\mathbb{Z} / i)^{n}} \varphi(\mathbf{a}) \psi(\mathbf{a})
$$

Let $\bar{\theta}_{i \Delta}=\frac{1}{E_{\Delta}(i)} \sum_{\mathbf{a} \in \Delta \cap(\mathbb{Z} / i)^{n}} \theta_{\Delta}\left(\frac{\mathbf{a}}{i}\right)$. We define $d_{i \Delta}, \tilde{\theta}_{i \Delta} \in W(i \Delta)$ by

$$
d_{i \Delta}(\mathbf{a})=1, \tilde{\theta}_{i \Delta}(\mathbf{a})=\frac{\theta_{\Delta}(\mathbf{a})-\bar{\theta}_{i \Delta}}{i}, \quad \mathbf{a} \in \Delta \cap(\mathbb{Z} / i)^{n} .
$$

Theorem 1.9. $\left(X_{\Delta}, L_{\Delta}\right)$ is asymptotically relatively Chow semistable in the toric sense if there is an $i_{0}$ such that for each $i \geqslant i_{0}$, and there exists $s_{i}$, such that

$$
\sum_{\mathbf{a} \in \Delta \cap(\mathbb{Z} / i)^{n}} i \mathbf{a}+s_{i} \sum_{\mathbf{a} \in \Delta \cap(\mathbb{Z} / i)^{n}} \tilde{\theta}_{i \Delta}(\mathbf{a}) \mathbf{a}=\frac{i E_{\Delta}(i)}{\operatorname{Vol}(\Delta)} \int_{\Delta} \mathbf{x} d x
$$

and

$$
\frac{i^{n}(n+1) ! \operatorname{Vol}(\Delta)}{E_{\Delta}(i)}\left(d_{i \Delta}+s_{i} \tilde{\theta}_{i \Delta}\right) \in \operatorname{Ch}(i \Delta),
$$

where $\operatorname{Ch}(i \Delta) \subset W(i \Delta)$ is the Chow polytope of $\left(X_{\Delta}, L_{\Delta}^{i}\right)$. Furthermore, it is asymptotically relatively Chow stable in the toric sense if

$$
\frac{i^{n}(n+1) ! \operatorname{Vol}(\Delta)}{E_{\Delta}(i)}\left(d_{i \Delta}+s_{i} \tilde{\theta}_{i \Delta}\right) \in \operatorname{Int}(\operatorname{Ch}(i \Delta)) .
$$

In [35], Ross-Thomas gave a description of Chow stability by using the Hilbert-Mumford criterion for the $\mathbb{C}^{*}$-actions induced by test configurations [12]. Inspired by this idea, we give an alternative reduction of the relative Chow stability of toric manifolds in Section 4. In order to see its relation to relative $K$-stability, we define

$$
\mathcal{Q}_{\Delta}(i, g)=E_{\Delta}(i) \int_{\Delta} g d x-\operatorname{Vol}(\Delta) \sum_{\mathbf{a} \in \Delta \cap(\mathbb{Z} / i)^{n}}\left(1+s_{i} \tilde{\theta}_{i \Delta}(\mathbf{a})\right) g(\mathbf{a}) \geqslant 0
$$

for $g \in P L(\Delta, i)$. Here $P L(\Delta, i)$ is a subset of piecewise linear concave functions (see Section 3.2). One can see that the Chow weight coincides with $\mathcal{Q}_{\Delta}(i, \cdot)$. 
TABLE 1. Relative stability in the toric sense of toric Fano threefolds

\begin{tabular}{|c|c|c|}
\hline Notation & Relative $K$-stability & $\begin{array}{l}\text { Asymptotic relative Chow stability } \\
\text { (Definition } 1.9 \text { ) }\end{array}$ \\
\hline $\mathbb{C} P^{3}$ & stable & stable \\
\hline $\mathcal{B}_{1}$ & unstable & unstable \\
\hline $\mathcal{B}_{2}$ & stable & \\
\hline $\mathcal{B}_{3}$ & stable & \\
\hline $\mathcal{B}_{4}$ & $\underline{\text { stable }}$ & stable \\
\hline $\mathcal{C}_{1}$ & stable & \\
\hline $\mathcal{C}_{2}$ & unstable & unstable \\
\hline $\mathcal{C}_{3}$ & $\underline{\text { stable }}$ & $\underline{\text { stable }}$ \\
\hline $\mathcal{C}_{4}$ & stable & \\
\hline $\mathcal{C}_{5}$ & stable & stable \\
\hline $\mathcal{D}_{1}$ & unstable & unstable \\
\hline $\mathcal{D}_{2}$ & unstable & unstable \\
\hline $\mathcal{E}_{1}$ & unstable & unstable \\
\hline $\mathcal{E}_{2}$ & unstable & unstable \\
\hline $\mathcal{E}_{3}$ & stable & \\
\hline $\mathcal{E}_{4}$ & stable & unstable \\
\hline $\mathcal{F}_{1}$ & $\underline{\text { stable }}$ & $\underline{\text { stable }}$ \\
\hline $\mathcal{F}_{2}$ & stable & \\
\hline
\end{tabular}

(1) All stable in the table are known before. Others are new in the present paper. All

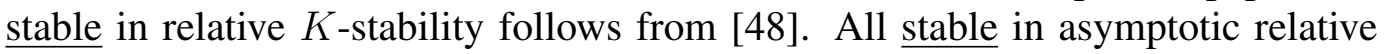
Chow stability (Definition 1.9] follows from [24] and [18].

(2) Other relative $K$-stability/instability follows from Thereom 1.4.(Proposition 5.1).

(3) Asymptotic relative Chow unstability except for $\mathcal{E}_{4}$ follows from Theorem 1.11

(4) Asymptotic relative Chow unstability of $\mathcal{E}_{4}$ follows from Proposition 5.5

Theorem 1.10. For any $i \in \mathbb{Z}_{+},\left(X_{\Delta}, L_{\Delta}^{i}\right)$ is relatively Chow semistable in the toric sense if and only if $\mathcal{Q}_{\Delta}(i, g) \geqslant 0$, for all $g \in P L(\Delta, i)$, where $s_{i}$ is the constant in Theorem 1.9 . In addition, it is relatively Chow stable in the toric sense if the equality holds only if $g$ is an affine linear function.

We would like to point out that Theorems 1.4, 1.9, 1.10 also hold for general polarized toric varieties. Concerning on relation between Chow and $K$-stabilities, we have:

Theorem 1.11. If a polarized toric manifold $(X, L)$ is asymptotically relatively Chow semistable in the toric sense, then it is relatively $K$-semistable in the toric sense.

In view of Theorem 1.5 , we also have the following.

Corollary 1.12. If $X$ is one of $\mathcal{B}_{1}, \mathcal{C}_{2}, \mathcal{D}_{1}, \mathcal{D}_{2}, \mathcal{E}_{1}$ and $\mathcal{E}_{2}$, then $X$ is asymptotically relatively Chow unstable. 
In general, asymptotic Chow semistability is much stronger than $K$-semistability. In order to see the direct evidence of the difference between Chow stability and $K$-stability consider the first counter-example that was discovered in [34]. They used the non-symmetric Kähler-Einstein toric Fano 7-fold of [29]. In the case where $X$ is non-toric, lower dimensional counter-examples were discovered by Odaka [31] and Vedova and Zuddas [42]. In [29], it was also proved that all toric Fano manifolds with the vanishing Futaki invariant are symmetric if $\operatorname{dim} X \leqslant 6$. Note that if $X$ is a symmetric toric Fano manifold, then $\left(X,-K_{X}\right)$ is asymptotically Chow stable [24, 15]. Hence the lowest dimension for an anti-canonically polarized Kähler-Einstein toric Fano manifold $\left(X,-K_{X}\right)$ to be asymptotically Chow unstable is 7 . One aim of this paper is to provide such an example in a lower dimensional toric case. We have found a 3-dimensional toric orbifold example admitting the Kähler-Einstein metric but which is asymptotically Chow unstable in the case where $X$ is $\mathbb{Q}$-Fano (Proposition 5.3). When we consider the relative stabilities, we find the smooth example $\mathcal{E}_{4}$ which is relatively $K$-stable but not asymptotically relatively Chow semistable (Proposition 5.5). The asymptotic Chow stability of $\mathbb{C} P^{3}, \mathcal{B}_{4}, \mathcal{C}_{3}, \mathcal{C}_{5}, \mathcal{F}_{1}$ follows from [18, 24]. Hence, we list all the determined stability of toric Fano threefolds in this paper in Table 1. Note that the stabilities are all in the toric sense. It is an interesting question to complete the table, i.e. to determine the remaining stabilities.

This paper is organized as follows. Section 2 is a brief review of toric varieties and the reduction of relative $K$-stability on toric manifolds which will be used at later stages in the paper. We also prove Theorem 1.4. In Sections 3 and 4 we shall discuss the two ways of reduction of the relative Chow stability on toric manifolds. In Section 5, we present various examples for the stabilities considered in the paper. We compute normalized potentials on toric Fano threefolds and verify the relative $K$-stability or instablity in Section 5.1 . We also provide an example of $K$-stable toric Fano orbifold $X$ which is asymptotically Chow unstable in $\operatorname{dim} X=3$. Finally, we discuss the asymptotic relative Chow stability of toric Fano threefolds. The computational results for $\theta_{\Delta}$ and $\Delta^{-}$are listed in Table 2.

Acknowledgements. The first author would like to thank Professors Y. Nakagawa, Y. Sano and A. Higashitani for their valuable comments and discussions. In particular, Higashitani suggested to us to use the toric package [4] for our computations.

\section{PRELIMINARIES}

2.1. Toric varieties. We review some of notations of toric varieties. Detailed discussion on the general theory can be found in [8]. Let $\mathfrak{M}$ be a lattice of rank $n$, where $\mathfrak{N}=$ $\operatorname{Hom}(\mathfrak{M}, \mathbb{Z})$ is the $\mathbb{Z}$-dual of $\mathfrak{M}$. We define $\mathfrak{M}_{\mathbb{R}}:=\mathfrak{M} \otimes_{\mathbb{Z}} \mathbb{R} \cong{ }^{n}$ (resp. $\mathfrak{N}_{\mathbb{R}}:=\mathfrak{N} \otimes_{\mathbb{Z}} \mathbb{R}$ ). Let $\Sigma$ denote a complete fan in $\mathfrak{N}_{\mathbb{R}}$, i.e. $\cup_{\sigma \in \Sigma} \sigma=\mathfrak{N}_{\mathbb{R}}$. The $k$-dimensional cones of $\Sigma$ form a set $\Sigma(k)$. Let $\sigma$ be a cone in $\Sigma$. The associated affine scheme $U_{\sigma}:=\operatorname{Spec} \mathbb{C}\left[\mathfrak{M} \cap \sigma^{\vee}\right]$ is called an affine toric variety. Then $\Sigma$ defines a toric variety $X:=X(\mathfrak{N}, \Sigma)$ by constructing the disjoint union of the affine toric varieties $U_{\sigma}$, where one glues $U_{\sigma_{1}}$ and $U_{\sigma_{2}}$ along the open subvariety $U_{\sigma_{1} \cap \sigma_{2}}$, for $\sigma_{1}, \sigma_{2} \in \Sigma$. We generally define a toric variety $X$ as a complex algebraic normal variety containing a torus $T_{\mathbb{C}}^{n}=\operatorname{Spec} \mathbb{C}[\mathfrak{M}]$ as a Zariski open subset, such that, the action of $T_{\mathbb{C}}^{n}$ on itself extends to an algebraic action of $T_{\mathbb{C}}^{n}$ on $X$.

A polytope $\Delta \subseteq \mathfrak{M}_{\mathbb{R}}$ is called a lattice (resp. rational) polytope if all its vertices are in $\mathfrak{M}$ (resp. $\mathfrak{M}_{\mathbb{Q}}=\mathfrak{M} \otimes_{\mathbb{Z}} \mathbb{Q}$ ). Let $\Delta \subseteq \mathfrak{M}_{\mathbb{R}}$ be a rational $n$-dimensional polytope with 
$0 \in \operatorname{Int} \Delta$. We define the dual polytope $\Delta^{\circ} \subseteq \mathfrak{N}_{\mathbb{R}}$ by

$$
\Delta^{\circ}:=\left\{a \in \mathfrak{N}_{\mathbb{R}} \mid\langle a, b\rangle \geqslant-1 \text { for all } b \in \Delta\right\},
$$

which is also a rational $n$-dimensional polytope with $0 \in \operatorname{Int} \Delta^{\circ}$. We denote a face $F$ of $\Delta^{\circ}$ by $F \prec \Delta^{\circ}$. The fan $\mathcal{N}_{\Delta}:=\left\{\operatorname{pos}(F) \mid F \prec \Delta^{\circ}\right\}$ is called the normal fan of $\Delta$, where $\operatorname{pos}(F)$ is the linear positive hull of $F$. For a rational polytope $\Delta \subseteq \mathfrak{M}_{\mathbb{R}}$, we define the associated toric variety by $X_{\Delta}:=X\left(\mathfrak{N}, \mathcal{N}_{\Delta}\right)$. In particular, when $\Delta$ satisfies Delzant's condition, it corresponds to a smooth compact toric manifold. It is well-known that there is the bijective correspondence between $n$-dimensional lattice polytopes and compact toric varieties with $\left(\mathbb{C}^{*}\right)^{n}$-equivariant very ample line bundles.

The discussion on examples (Section 5) will focus on toric Fano threefolds. We recall the related notations here. See [10] and [28] for more details.

Let $X$ be a complex projective normal variety. We call $X$ a $\mathbb{Q}$-Fano variety if the anticanonical divisor $-K_{X}$ is an ample $\mathbb{Q}$-Cartier divisor. Furthermore, $X$ is Fano variety if $-K_{X}$ is an ample Cartier divisor. Let $P \subseteq \mathfrak{N}_{\mathbb{R}}$ be an $n$-dimensional lattice polytope which contains the origin in its interior. Then $P$ is called a canonical Fano polytope if $\operatorname{Int} P \cap \mathfrak{N}=$ $\{0\}$. Furthermore, $P$ is called a Fano polytope if the vertices of any facet of $P$ form a $\mathbb{Z}$-basis of the lattice $\mathfrak{N}$. By [28, Proposition 2.3.7], there is a bijective correspondence between isomorphism classes of Fano polytopes (resp. canonical Fano polytopes) and smooth toric Fano varieties (resp. toric Fano varieties with canonical singularities). Here and hereafter we assume that all singularities are at worst orbifold singularities. Hence they are associated to complete simplicial fans [8, Theorem 3.1.19].

Let $X$ be a complex normal variety. Recall that $X$ is called $\mathbb{Q}$-factorial if any Weil divisor is $\mathbb{Q}$-Cartier. For the toric case, we have a well-known description in terms of a Fano polytope. A polytope is called simplicial if any facet is a simplex. It was shown that simplicial Fano polytopes correspond uniquely up to isomorphism to $\mathbb{Q}$-factorial toric Fano varieties (see Proposition 2.3.12 in [28]). Since a toric variety $X$ has only finite quotient singularities (that is, $X$ is an orbifold) if and only if the associated fan $\Sigma$ is simplicial, $\mathbb{Q}$-factorial toric Fano varieties are toric Fano orbifolds.

In [22], Kasprzyk found that there are 12,190 $\mathbb{Q}$-factorial toric Fano varieties up to isomorphism. In the case when $X$ is smooth, toric Fano threefolds are classified in [1, 43]. There are 18 toric Fano threefolds, that is, $\mathbb{C} P^{3}, \mathcal{B}_{1}, \mathcal{B}_{2}, \mathcal{B}_{3}, \mathcal{B}_{4}=\mathbb{C} P^{2} \times \mathbb{C} P^{1}, \mathcal{C}_{1}, \mathcal{C}_{2}$, $\mathcal{C}_{3}=\mathbb{C} P^{1} \times \mathbb{C} P^{1} \times \mathbb{C} P^{1}, \mathcal{C}_{4}=\mathbb{C} P^{1} \times\left(\mathbb{C} P^{2} \# \overline{\mathbb{C} P^{2}}\right), \mathcal{C}_{5}=\mathbb{C} P_{\mathbb{C} P^{1} \times \mathbb{C} P^{1}}(\mathcal{O} \oplus \mathcal{O}(1,-1))$, $\mathcal{D}_{1}, \mathcal{D}_{2}, \mathcal{E}_{1}, \mathcal{E}_{2}, \mathcal{E}_{3}=\mathbb{C} P^{1} \times\left(\mathbb{C} P^{2} \# 2 \overline{\mathbb{C} P^{2}}\right), \mathcal{E}_{4}, \mathcal{F}_{1}=\mathbb{C} P^{1} \times\left(\mathbb{C} P^{2} \# 3 \overline{\mathbb{C} P^{2}}\right)$ and $\mathcal{F}_{2}$. Note that we use the same notation as in [1]. These classification results are available online at [49, 30].

2.2. Reduction of relative $K$-stability. In this subsection, we recall the reduction of the Futaki invariant on toric manifolds. We also present the formulae to determine the normalized potential $\theta_{\Delta}$ of the extremal vector field in symplectic coordinate and criterions for relative $K$-stability and instablity.

First we recall the Futaki invariant and the extremal vector field. Let $\left(X, \omega_{g}\right)$ be a compact Kähler manifold. Let $\operatorname{Aut}^{0}(X)$ be the identity component of the biholomorphisms group of $X$. Then $\operatorname{Aut}^{0}(X)$ has a semidirect decomposition

$$
\operatorname{Aut}^{0}(X)=\operatorname{Aut}_{r}(X) \ltimes R_{u},
$$


where $\operatorname{Aut}_{r}(X)$ is a reductive algebraic subgroup of $\operatorname{Aut}^{0}(X)$, which is the complexification of a maximal compact subgroup $K$, and $R_{u}$ is the nilpotent radical of $\operatorname{Aut}^{0}(X)$. We denote the Lie algebra of $\operatorname{Aut}_{r}(X)$ by $\eta_{r}(X)$. Let $v \in \eta_{r}(X)$ so that its imaginary part generates a one-parameter compact subgroup of $K$. Then if the Kähler form $\omega_{g}$ is $K$-invariant, there exists a unique real-valued function $\theta_{v}\left(\omega_{g}\right)$ (called normalized potential of $v$ ) such that

$$
i_{v} \omega_{g}=\sqrt{-1} \bar{\partial} \theta_{v}\left(\omega_{g}\right) \text { and } \int_{X} \theta_{v}\left(\omega_{g}\right) \frac{\omega_{g}^{n}}{n !}=0 .
$$

For simplicity, we denote the set of such potentials $\theta_{v}$ by $\Xi_{\omega_{g}}$. Then the Futaki invariant on $\eta_{r}$ can be written as

$$
F(v)=-\int_{X} \theta_{v}\left(\omega_{g}\right)\left(S\left(\omega_{g}\right)-\bar{S}\right) \frac{\omega_{g}^{n}}{n !},
$$

where $S\left(\omega_{g}\right)$ is the scalar curvature of $\omega_{g}$. In [17], Futaki and Mabuchi defined the extremal vector field, $V=g^{i \bar{j}}\left(\operatorname{proj}\left(S\left(\omega_{g}\right)\right)\right)_{\bar{j}} \frac{\partial}{\partial z_{i}}$ in $\eta_{r}(X)$ for the Kähler class $\left[\omega_{g}\right]$, where $\operatorname{proj}\left(S\left(\omega_{g}\right)\right)$ is the $L^{2}$-inner projection of the scalar curvature of $\omega_{g}$ to $\Xi_{\omega_{g}}$. They showed that $V$ is independent of the choice of $K$-invariant metrics in $\left[\omega_{g}\right]$, and its potential is uniquely determined as the dual of the Futaki invariant with respect to the $L^{2}$ bilinear form

$$
F(v)=-\int_{X} \theta_{v}\left(\omega_{g}\right) \theta_{V}\left(\omega_{g}\right) \frac{\omega_{g}^{n}}{n !}, \forall v \in \eta_{r}(X)
$$

Now we consider the reduction on a polarized toric manifolds $(X, L)$. Choose an $\left(S^{1}\right)^{n}$ invariant Kähler metric $g$ with $\omega_{g} \in 2 \pi c_{1}(L)$. By choosing a base point, we identify the open dense orbit of the complex torus action on $X$ with $\left(\mathbb{C}^{*}\right)^{n}$ and use the coordinates $\left(z_{1}, \ldots, z_{n}\right)$ induced from $\left(\mathbb{C}^{*}\right)^{n}$. Denote the affine logarithmic coordinates $w_{i}=\log z_{i}=$ $y_{i}+\sqrt{-1} \eta_{i}$. Then $\omega_{g}$ is determined by a smooth convex function $\varphi$ which depends only on $y_{1}, \ldots, y_{n} \in \mathbb{R}^{n}$ in the coordinates $\left(w_{1}, \ldots \ldots, w_{n}\right)$, namely

$$
\omega_{g}=2 \sqrt{-1} \partial \bar{\partial} \varphi
$$

on $\left(\mathbb{C}^{*}\right)^{n}$. As is well-known, the moment map can be given by $D \varphi$ and the image $\Delta=$ $D \varphi\left(\mathbb{R}^{n}\right)$ is a polytope. We denote by $x_{i}=\frac{\partial \varphi}{\partial y_{i}}, i=1, \ldots, n$, the symplectic coordinates. Note that in the affine logarithm coordinates $\left(w_{1}, \ldots, w_{n}\right),\left\{\frac{\partial}{\partial w_{i}}, i=1, \ldots, n\right\}$ is a basis of the Lie algebra of $T_{\mathbb{C}}^{n}$ as a complex subalgebra of the Lie algebra of holomorphic vector fields on $X$. The following lemma was given in [47] on how to determine $\theta_{V}\left(\omega_{g}\right)$ in symplectic coordinates through the Futaki invariant.

Lemma 2.1. Let $g$ be an $\left(S^{1}\right)^{n}$-invariant metric on $X$. Assume $V$ is the extremal vector field and $\theta_{V}\left(\omega_{g}\right)$ is the normalized potential associated to $\omega_{g}$ by (2.1). Then there are $2 n$-numbers $a_{i}$ and $c_{i}$ such that

$$
\theta_{V}\left(\omega_{g}\right)=\sum_{i=1}^{n} a_{i}\left(x_{i}+c_{i}\right)=: \theta_{\Delta}
$$


where $x=\left(x_{i}\right)=D \varphi \in \Delta$. Moreover $a_{i}$ and $c_{i}$ are determined uniquely by $2 n$-equations,

$$
\begin{aligned}
& \frac{1}{(2 \pi)^{n}} F\left(\frac{\partial}{\partial w_{i}}\right)=-\int_{\Delta}\left(\sum_{j=1}^{n} a_{j}\left(x_{j}+c_{j}\right)\right)\left(x_{i}+c_{i}\right) d x, i=1, \ldots, n, \\
& \int_{\Delta}\left(x_{i}+c_{i}\right) d x=0, i=1, \ldots, n .
\end{aligned}
$$

As mentioned in the introduction, the relative $K$-stability in the toric sense refers to the positivity of the linear functional $(1.2)$ for convex functions. Note that $\mathcal{L}_{\Delta}(u)$ is invariant when adding an affine linear function to $u$. Without loss of generality, we assume 0 lies in the interior of $\Delta$. Hence, it suffices to consider convex functions normalized at $\mathbf{0}$ in the sense that $\inf _{x \in \Delta} u(x)=u(\mathbf{0})=0$. When $X$ is a toric Fano manifold, it is observed in [47] that

$$
\mathcal{L}_{\Delta}(u)=\int_{\Delta}\left(\sum_{i=1}^{n} x_{i} u_{i}-u\right)+\left(1-\theta_{\Delta}\right) u d x
$$

for $C^{1}$ functions by an integration by parts from (1.2). Here $u_{i}=\frac{\partial u}{\partial x_{i}}$. By approximation, it is easy to see that (2.7) can also be used for the computation of $\mathcal{L}_{\Delta}(u)$ for piecewise $C^{1}$ functions. As can be seen from (2.7), the positivity of $\mathcal{L}_{\Delta}$ relies heavily on the positivity of $1-\theta_{\Delta}$. Assume $\theta_{\Delta}=\sum_{i=1}^{n} a_{i} x_{i}+c$. Then we shall prove Theorem 1.4

Proof of Theorem 1.4 (1) is obvious [47]. We only need to consider (2). If $1-\theta_{\Delta}<0$, i.e. $\Delta^{-}=\Delta$, it is obvious that all simple piecewise linear convex functions of the form $\max \left\{\sum_{i=1}^{n} b_{i} x_{i}, 0\right\}$ will destabilize $\Delta$. So we assume $1-\theta_{\Delta}=0$ intersects the interior of $\Delta$. Let

$$
u=\max \left\{-\left(1-\theta_{\Delta}\right), 0\right\}
$$

Then

$$
\sum_{i=1}^{n} x_{i} u_{i}-u= \begin{cases}1-c, & x \in \Delta^{-} \\ 0, & x \in \Delta \backslash \Delta^{-}\end{cases}
$$

Hence,

$$
\mathcal{L}_{\Delta}(u)=(1-c) \operatorname{Vol}\left(\Delta^{-}\right)-\int_{\Delta^{-}}\left(1-\theta_{\Delta}\right)^{2} d x .
$$

The theorem follows.

The condition in this theorem is not sharp but we will see in Section 5 that this criterion can determine all the stable toric Fano threefolds.

\section{RELATIVE CHOW STABILITY OF TORIC MANIFOLDS}

In this section, we consider relative Chow stability of polarized toric manifolds. 
3.1. Notions of Chow stabilities. We first recall various notions of Chow stabilities. We refer to the monograph [16] by Futaki for a more general concept of Chow stability in Kähler geometry. In [23, 25], Mabuchi defined the notion of relative Chow stability in order to consider the existence problem of extremal Kähler metrics. A historical background of relative GIT stability is given by Székelyhidi [38, Chapter 1].

Let $G$ be a connected reductive complex algebraic group with Lie algebra $\mathfrak{g}$. Let $\mathbf{V}$ be a finite dimensional complex vector space. Suppose that $G$ acts linearly on V. Assume $v^{*}$ is a nonzero vector in $\mathbf{V}$ which is a representative of $v=\left[v^{*}\right] \in \mathbb{P}(\mathbf{V})$. According to GIT, $v^{*}$ is $G$-semistable if the closure of the $G$-orbit $\mathcal{O}_{G}\left(v^{*}\right)$ does not contain the origin. Furthermore, $v^{*}$ is $G$-stable if $\mathcal{O}_{G}\left(v^{*}\right)$ is closed. We call $v^{*} G$-unstable if $v^{*}$ is not $G$ semistable. Analogously, $v \in \mathbb{P}(\mathbf{V})$ is said to be $G$-semistable (resp. stable, unstable) if any representative of $v$ is $G$-semistable (resp. stable, unstable).

To feature relative stability, following [38, Chapter 1], we consider a torus $T$ in $G$, and denote its Lie algebra by $\mathfrak{t}$. Then we define subalgebras of $\mathfrak{g}$ by

$$
\begin{aligned}
\mathfrak{g}_{T} & =\{\alpha \in \mathfrak{g} \mid[\alpha, \beta]=0 \text { for all } \beta \in \mathfrak{t}\}, \\
\mathfrak{g}_{T^{\perp}} & =\left\{\alpha \in \mathfrak{g}_{T} \mid\langle\alpha, \beta\rangle=0 \text { for all } \beta \in \mathfrak{t}\right\},
\end{aligned}
$$

where $\langle\cdot, \cdot\rangle$ is a rational invariant inner product. We denote the image of $\mathfrak{g}_{T}$ (resp. $\mathfrak{g}_{T^{\perp}}$ ) under the exponential map by $G_{T}$ (resp. $G_{T^{\perp}}$ ).

Definition 3.1. [38] Let $T$ be a torus in $G$ fixing the point $v$. Then $v$ is said to be semistable (resp. stable, unstable) relative to $T$ if it is $G_{T^{\perp}}$-semistable (resp. stable, unstable).

The Hilbert-Mumford criterion says that $v \in \mathbb{P}(\mathbf{V})$ is $G$-semistable if and only if $v$ is $H$ semistable for any maximal algebraic torus $H \subset G$ [9, p.137]. When $G$ itself is isomorphic to an algebraic torus, the above stabilities can be described by the weight polytopes of the actions as follows. Let $\chi(G)$ denote the character group of $G$. Then $\chi(G)$ consists of algebraic homomorphisms $\chi: G \longrightarrow \mathbb{C}^{*}$. If we fix an isomorphism $G \cong\left(\mathbb{C}^{*}\right)^{N+1}$, we may express each $\chi$ as a Laurent monomial

$$
\chi\left(t_{1}, \ldots, t_{N+1}\right)=t_{1}^{a_{1}} \cdots t_{N+1}^{a_{N+1}}, \quad t_{i} \in \mathbb{C}^{*}, a_{i} \in \mathbb{Z} .
$$

Thus, there is the identification between $\chi(G)$ and $\mathbb{Z}^{N+1}$ by $\chi=\left(a_{1}, \ldots, a_{N+1}\right) \in \mathbb{Z}^{N+1}$. Then it is well-known that $\mathbf{V}$ decomposes under the action of $G$ into weight spaces

$$
\mathbf{V}=\bigoplus_{\chi \in \chi(G)} \mathbf{V}_{\chi}, \quad \mathbf{V}_{\chi}:=\left\{v^{*} \in \mathbf{V} \mid t \cdot v^{*}=\chi(t) \cdot v^{*}, t \in G\right\}
$$

Definition 3.2. Let $v^{*}$ be a nonzero vector in $\mathbf{V}$ with $v^{*}=\sum_{\chi \in \chi(G)} v_{\chi}, v_{\chi} \in \mathbf{V}_{\chi}$. The weight polytope of $v^{*}$ (with respect to $G$-action) is the convex lattice polytope in $\chi(G) \otimes$ $\mathbb{R} \cong \mathbb{R}^{N+1}$ defined by

$$
\mathcal{N}_{G}\left(v^{*}\right):=\operatorname{Conv}\left\{\chi \in \chi(G) \mid v_{\chi} \neq 0\right\} \subseteq \mathbb{R}^{N+1} .
$$

According to [9, Theorem 9.2], $v^{*}$ is $G$-semistable (resp. stable) if and only if $0 \in$ $\mathcal{N}_{G}\left(v^{*}\right)$ (resp. $0 \in \operatorname{Int} \mathcal{N}_{G}\left(v^{*}\right)$ ). In the relative stability setting, we also have the following.

Proposition 3.3. [38, Theorem 1.5.2] Let $T$ be a torus in G fixing the point $v$. $v$ is semistable (resp. stable) relative to $T$ if and only if the orthogonal projection of the origin onto the minimal affine subspace containing $\mathcal{N}_{G}\left(v^{*}\right)$ is in $\mathcal{N}_{G}\left(v^{*}\right)\left(\operatorname{resp}, \operatorname{relint} \mathcal{N}_{G}\left(v^{*}\right)\right)$. 
Next we define Chow form and Chow stability of irreducible projective varieties. See [19. 46] for more details. Let $X \subset \mathbb{C} P^{N}$ be an $n$-dimensional irreducible complex projective variety of degree $d$. Recall that the Grassmann variety $\mathbb{G}\left(k, \mathbb{C} P^{N}\right)$ parameterizes $k$-dimensional projective linear subspaces of $\mathbb{C} P^{N}$. The associated hypersurface of $X \subset \mathbb{C} P^{N}$ is the subvariety in $\mathbb{G}\left(N-n-1, \mathbb{C} P^{N}\right)$ which is given by

$$
Z_{X}:=\left\{W \in \mathbb{G}\left(N-n-1, \mathbb{C} P^{N}\right) \mid W \cap X \neq \emptyset\right\} .
$$

It is known that is $Z_{X}$ is an irreducible hypersurface with $\operatorname{deg} Z_{X}=d$ in the Plücker coordinates. In particular, $Z_{X}$ is given by the vanishing of a section $R_{X}^{*} \in H^{0}(\mathbb{G}(N-n-$ $\left.\left.1, \mathbb{C} P^{N}\right), \mathcal{O}(d)\right)$. We call $R_{X}^{*}$ the Chow form of $X$. Note that $R_{X}^{*}$ is well defined up to a multiplicative constant. Let $\mathbf{V}:=H^{0}\left(\mathbb{G}\left(N-n-1, \mathbb{C} P^{N}\right), \mathcal{O}(d)\right)$ and $R_{X} \in \mathbb{P}(\mathbf{V})$ be the projectivization of $R_{X}^{*}$. We call $R_{X}$ the Chow point of $X$. The weight polytope of $R_{X}^{*} \in \mathbf{V}$ with respect to the action $\left(\mathbb{C}^{*}\right)^{N+1} \subset \mathrm{GL}(N+1, \mathbb{C})$ of diagonal matrices is called Chow polytope of $X$, and is denoted by $\mathrm{Ch}(X)$. See [19, Chapter 6] for more details. Since we have the natural action of $G=\mathrm{SL}(N+1, \mathbb{C})$ into $\mathbb{P}(\mathbf{V})$, we can define stabilities of $R_{X}$ as follows.

Definition 3.4. Let $X \subset \mathbb{C} P^{N}$ be an irreducible, $n$-dimensional complex projective variety. Then $X$ is said to be Chow semistable (resp. stable, unstable) if the Chow point $R_{X}$ of $X$ is $\mathrm{SL}(N+1, \mathbb{C})$-semistable (resp. stable, unstable).

We consider relative Chow stability when the Futaki invariant does not vanish. Choose $T=\beta$ to be the $\mathbb{C}^{*}$ - action induced by the extremal vector field $V . T$ also acts on $\mathbb{P}(\mathbf{V})$.

Definition 3.5. Let $X \subset \mathbb{C} P^{N}$ be an irreducible, $n$-dimensional complex projective variety. Then $X$ is said to be relatively Chow semistable (resp. stable, unstable) if the Chow point $R_{X}$ of $X$ is $\mathrm{SL}(N+1, \mathbb{C})$-semistable (resp. stable, unstable) relative to $T$.

Definition 3.6. Let $(X, L)$ be a polarized variety. For $i \gg 0$, let $\Psi_{i}: X \longrightarrow \mathbb{P}\left(H^{0}\left(X, L^{i}\right)^{*}\right)$ be the Kodaira embedding.

(1) Suppose that $L$ is very ample. $(X, L)$ is said to be relatively Chow semistable (resp. stable, unstable) if $\Psi_{1}(X) \subset \mathbb{P}\left(H^{0}(X, L)^{*}\right)$ is relatively Chow semistable (resp. stable, unstable).

(2) $(X, L)$ is called asymptotically relatively Chow semistable (resp. stable) if there is an $i_{0}$ such that $\Psi_{i}(X)$ is relatively Chow semistable (resp. stable) for each $i \geqslant i_{0}$.

We say that $(X, L)$ is asymptotically relatively Chow unstable if it is not asymptotically relatively Chow semistable.

3.2. Reduction on toric manifolds. We reduce the relative Chow stability of polarized toric manifolds by developing on an idea in [32, 33].

Recall that $T_{\Delta}^{\mathbb{C}}$ of $\mathrm{SL}(N+1, \mathbb{C})$ is given by

$$
\begin{aligned}
T_{\Delta}^{\mathbb{C}} & \hookrightarrow G=\left(\mathbb{C}^{*}\right)^{N+1} \cap \operatorname{SL}(N+1, \mathbb{C}) \\
\left(t_{1}, \ldots, t_{N}\right) & \longmapsto\left(t_{1}, \ldots, t_{N},\left(t_{1} \cdots t_{N}\right)^{-1}\right) .
\end{aligned}
$$

In particular, $T_{\Delta}^{\mathbb{C}} \cong\left(\mathbb{C}^{*}\right)^{N}$. We view the Lie algebra of $T_{\Delta}^{\mathbb{C}}$ as a subalgebra of $\operatorname{sl}(N+$ $1, \mathbb{C})$ by considering the traceless part. The inner product $\langle$,$\rangle on \operatorname{sl}(N+1, \mathbb{C})$ is given by 
$\langle A, B\rangle=\operatorname{Tr}(A B)$. Let $\left\{\mathbf{a}_{1}, \ldots, \mathbf{a}_{N+1}\right\}$ be all the lattice points in $\Delta$. We define

$$
\bar{\theta}_{\Delta}=\frac{1}{N+1} \sum_{j=1}^{N+1} \theta_{\Delta}\left(\mathbf{a}_{j}\right)
$$

Let $\theta_{\Delta}$ be the potential function as in Section 2 , and $T=\beta$ be the $\mathbb{C}^{*}$ - action induced by $V$. Then $T$ is given in $T_{\Delta}^{\mathbb{C}}$ by

$$
\begin{aligned}
T: \mathbb{C}^{*} & \hookrightarrow G \\
t & \longmapsto\left(t^{\left(\theta_{\Delta}\left(\mathbf{a}_{1}\right)-\bar{\theta}_{\Delta}\right)}, \ldots, t^{\left(\theta_{\Delta}\left(\mathbf{a}_{N+1}\right)-\bar{\theta}_{\Delta}\right)}\right) .
\end{aligned}
$$

Let $\operatorname{Ch}(\Delta)$ be the Chow polytope of $X_{\Delta} \subset \mathbb{C} P^{N}$. In the literature of Gelfand-KapranovZelevinsky's theory, $\operatorname{Ch}(\Delta)$ coincides with the Chow polytope [21]. In particular, it is known that the affine span of the secondary polytope is given by the following.

Proposition 3.7. [19, Chapter 7, Proposition 1.11] Let $\varphi=\left(\varphi_{1}, \ldots, \varphi_{N+1}\right)$ be a point in the affine hull of $\mathrm{Ch}(\Delta)$ in $\chi(G) \otimes \mathbb{R} \cong \mathbb{R}^{N+1}$. Then

$$
\sum_{j=1}^{N+1} \varphi_{j}=(n+1) ! \operatorname{Vol}(\Delta), \quad \sum_{j=1}^{N+1} \varphi_{j} \mathbf{a}_{j}=(n+1) ! \int_{\Delta} \mathbf{x} d x .
$$

Here $\mathbf{x}=\left(x_{1}, \ldots, x_{n}\right)$ and $\Delta \cap M=\left\{\mathbf{a}_{1}, \ldots, \mathbf{a}_{N+1}\right\}$ is all the lattice points in $\Delta$.

Denote

$$
d_{\Delta}=(1, \ldots, 1), \quad \tilde{\theta}_{\Delta}=\left(\left(\theta_{\Delta}\left(\mathbf{a}_{1}\right)-\bar{\theta}_{\Delta}\right), \ldots,\left(\theta_{\Delta}\left(\mathbf{a}_{N+1}\right)-\bar{\theta}_{\Delta}\right)\right)
$$

in $\chi(G) \otimes \mathbb{R}$. Then we have the following.

Theorem 3.8. $\left(X_{\Delta}, L_{\Delta}\right)$ is relatively Chow semistable in the toric sense if and only if there exists $s \in \mathbb{R}$ such that

$$
\sum_{j=1}^{N+1} \mathbf{a}_{j}+s \sum_{j=1}^{N+1} \tilde{\theta}_{\Delta}(\mathbf{a}) \mathbf{a}_{j}=\frac{N+1}{\operatorname{Vol}(\Delta)} \int_{\Delta} \mathbf{x} d x
$$

and

$$
\frac{(n+1) ! \operatorname{Vol}(\Delta)}{N+1}\left(d_{\Delta}+s \tilde{\theta}_{\Delta}\right) \in \operatorname{Ch}(\Delta) .
$$

Furthermore, it is relatively Chow stable in the toric sense if

$$
\frac{(n+1) ! \operatorname{Vol}(\Delta)}{N+1}\left(d_{\Delta}+s \tilde{\theta}_{\Delta}\right) \in \operatorname{Int}(\operatorname{Ch}(\Delta)) .
$$

Proof. We define a two dimensional subspace in $\mathbb{R}^{N+1}$ by $\mathbf{W}:=\operatorname{Span}_{\mathbb{R}}\left\{d_{\Delta}, \tilde{\theta}_{\Delta}\right\}$. Let $\beta_{1}, \ldots, \beta_{N-1} \in \mathbb{R}^{N+1}$ be a basis of the subspace perpendicular to $\mathbf{W}$. Note that $G_{T^{\perp}}$ is isomorphic to $\left(\mathbb{C}^{*}\right)^{N-1}$. Considering the projection

$$
\begin{aligned}
\pi_{G_{T^{\perp}}}: \chi(G) \otimes \mathbb{R} \cong \mathbb{R}^{N+1} \longrightarrow \chi\left(G_{T^{\perp}}\right) \otimes \mathbb{R} \cong \mathbb{R}^{N-1} \\
\varphi=\left(\varphi_{1}, \ldots, \varphi_{N+1}\right) \longmapsto\left(\left\langle\varphi, \beta_{1}\right\rangle, \ldots,\left\langle\varphi, \beta_{N-1}\right\rangle\right),
\end{aligned}
$$

we observe that $\mathcal{N}_{G_{T^{\perp}}}\left(R_{X_{\Delta}}\right)=\pi_{G_{T^{\perp}}}\left(\mathcal{N}_{G}\left(R_{X_{\Delta}}\right)\right) \subset \mathbb{R}^{N-1}$.

By definition, $R_{X_{\Delta}}$ is $G_{T^{\perp}}$-semistable if and only if $0 \in \mathcal{N}_{G_{T^{\perp}}}\left(R_{X_{\Delta}}\right)$. By the projection above, it is equivalent to $\mathbf{W} \cap \operatorname{Ch}(\Delta) \neq \emptyset$, that is, there exist $s_{1}, s_{2} \in \mathbb{R}$ such that 


$$
s_{1} d_{\Delta}+s_{2} \tilde{\theta}_{\Delta} \in \mathrm{Ch}(\Delta)
$$

By (3.2) and the fact $\sum_{j=1}^{N+1} \tilde{\theta}_{\Delta}\left(\mathbf{a}_{j}\right)=0$, we have $s_{1}=\frac{(n+1) ! \operatorname{Vol}(\Delta)}{N+1}$.

Remark 3.9. The reader should notice that (3.4) implies (3.3) by Proposition 3.7. This theorem extends Ono's description of Chow semistability [32, 33] to relative case.

Next, we consider asymptotic relative Chow semistability. Denote the Ehrhart polynomial of $\Delta$ by $E_{\Delta}(t)$. It has degree $n=\operatorname{dim} \Delta$ and satisfies

$$
E_{\Delta}(i)=\operatorname{Card}\left(i \Delta \cap \mathbb{Z}^{n}\right)=\operatorname{Card}\left(\Delta \cap(\mathbb{Z} / i)^{n}\right)
$$

for any positive integer $i \in \mathbb{Z}_{+}$. Moreover, $E_{\Delta}(t)$ has the form

$$
E_{\Delta}(t)=\operatorname{Vol}(\Delta) t^{n}+\frac{\operatorname{Vol}(\partial \Delta)}{2} t^{n-1}+\cdots+1
$$

by theorem of Ehrhart. Note that $\int_{i \Delta} \mathbf{x} d x=i^{n+1} \int_{\Delta} \mathbf{x} d x$, hence Theorem 1.9 follows from the same argument in the proof of Theorem 3.8 .

The asymptotic relative Chow semistability can be related to relative $K$-semistability in the toric sense. For this purpose, we recall some notations. For a fixed $\varphi \in W(i \Delta)$, let

$$
G_{\varphi}=\text { the convex hull of } \bigcup_{\mathbf{a} \in \Delta \cap(\mathbb{Z} / i)^{n}}\{(\mathbf{a}, t) \mid t \leqslant \varphi(\mathbf{a})\} \subset \mathfrak{M}_{\mathbb{R}} \times \mathbb{R} \cong \mathbb{R}^{n+1} .
$$

Then we define a piecewise linear function $g_{\varphi}: \Delta \longrightarrow \mathbb{R}$ by

$$
g_{\varphi}(\mathbf{x})=\max \left\{t \mid(\mathbf{x}, t) \in G_{\varphi}\right\} .
$$

The upper boundary of $G_{\varphi}$ can be regarded as the graph of $g_{\varphi}$. Furthermore, $g_{\varphi}$ has the following properties.

Lemma 3.10 ([19] p.221, Lemma 1.9). For any $\varphi \in W(i \Delta)$,

(a) the function $g_{\varphi}$ is concave.

(b) we have the equality

$$
\max \{\langle\varphi, \psi\rangle \mid \psi \in \mathrm{Ch}(i \Delta)\}=i^{n}(n+1) ! \int_{\Delta} g_{\varphi} d x .
$$

Denote $P L(\Delta, i)=\left\{g_{\varphi} \mid \varphi \in W(i \Delta)\right\}$. The proof of Theorem 1.10 is similar to [33]. One can see that the condition (1.6) holds if and only if the following condition holds:

$$
\max \{\langle\varphi, \psi\rangle \mid \psi \in \operatorname{Ch}(i \Delta)\} \geqslant \frac{i^{n}(n+1) ! \operatorname{Vol}(\Delta)}{E_{\Delta}(i)}\left\langle\varphi, 1+s_{i} \tilde{\theta}_{i \Delta}\right\rangle
$$

for all $\varphi \in W(i \Delta)$. By applying Lemma 3.10 to (3.7), we obtain (1.8).

Corollary 3.11. If $\left(X_{\Delta}, L_{\Delta}\right)$ is asymptotically relatively Chow semistable, then for any $i \in \mathbb{Z}_{+}$, there exists $s_{i}$ such that $(1.5)$ holds.

Proof. Applying (1.8) to affine linear functions, one can see that $\mathcal{Q}_{\Delta}(i, g)=0$. As in (3.9), $\mathcal{Q}_{\Delta}(i, g)$ can be written as a fractional polynomial in $i$. If (1.5) does not hold for an integer $i_{0} \in \mathbb{Z}_{+}$, then there is an integer $i_{1} \in \mathbb{Z}_{+}$such that $\left(X_{\Delta}, L_{\Delta}^{i}\right)$ is relatively Chow unstable for any $i>i_{1}$. 
Remark 3.12. (1) It is clear that $(1.5)$ is an over-determined linear system since there is only one parameter $s_{i}$, but $n$ equations. Hence, one can expect to find counter-examples from polytopes which are not symmetric with respect to $x_{1}, \ldots, x_{n}$. (Cf. Proposition 5.5). (2) When $V=0,1.5$ becomes

$$
\operatorname{Vol}(\Delta) s_{\Delta}(i)-E_{\Delta}(i) \int_{\Delta} \mathbf{x} d x=\mathbf{0}
$$

which is the necessary condition for $\left(X_{\Delta}, L_{\Delta}^{i}\right)$ to be Chow semistable proved by Ono[32, Theorem 1.4]. From the argument in the proof of Corollary 3.11, one can see that the following: let $\Delta \subseteq \mathfrak{M}_{\mathbb{R}}$ be a simple lattice polytope and $\left(X_{\Delta}, L_{\Delta}\right)$ be the associated polarized toric orbifold. If (3.8) does not fold for a positive integer $i_{0} \in \mathbb{Z}_{+}$, then there is a positive integer $i_{1} \in \mathbb{Z}_{+}$, such that $\left(X_{\Delta}, L_{\Delta}^{i}\right)$ is Chow unstable for any $i>i_{1}$.

Finally we show asymptotical relative Chow semistability implies relative $K$-semistability in the toric case. It is known that asymptotic Chow semistability implies $K$-semistability in general sense[35]. First, we need the following lemma.

Lemma 3.13. $s_{i}$ in 1.5 satisfies $s_{i}=-\frac{1}{2}+O\left(i^{-1}\right)$.

Proof. By $\int_{\Delta} \theta_{\Delta} d x=0$ and Lemma 3.3 of [47], we have

$$
\begin{aligned}
\bar{\theta}_{i \Delta} & =\frac{i^{n-1}}{2 E_{\Delta}(i)} \int_{\partial \Delta} \theta_{\Delta} d \sigma+O\left(i^{n-2}\right), \\
\sum_{\mathbf{a} \in \Delta \cap(\mathbb{Z} / i)^{n}} i \mathbf{a} & =i^{n+1} \int_{\Delta} \mathbf{x} d x+\frac{i^{n}}{2} \int_{\partial \Delta} \mathbf{x} d \sigma+O\left(i^{n-1}\right), \quad \text { and } \\
\sum_{\mathbf{a} \in \Delta \cap(\mathbb{Z} / i)^{n}} \theta_{V}(\mathbf{a}) \mathbf{a} & =i^{n} \int_{\Delta} \theta_{\Delta} \mathbf{x} d x+O\left(i^{n-1}\right) .
\end{aligned}
$$

Then (1.5) is written as

$$
\begin{aligned}
i E_{\Delta}(i) & \int_{\Delta} \mathbf{x} d x=\operatorname{Vol}(\Delta)\left(i^{n+1} \int_{\Delta} \mathbf{x} d x+\frac{i^{n}}{2} \int_{\partial \Delta} \mathbf{x} d \sigma+O\left(i^{n-1}\right)\right)+s_{i} \operatorname{Vol}(\Delta) \cdot \\
& {\left[i^{n} \int_{\Delta} \theta_{\Delta} \mathbf{x} d x+O\left(i^{n-1}\right)-\frac{i^{n} \int_{\Delta} \mathbf{x} d x+O\left(i^{n-1}\right)}{E_{\Delta}(i)}\left(\frac{i^{n-1}}{2} \int_{\partial \Delta} \theta_{\Delta} d \sigma+O\left(i^{n-2}\right)\right)\right] . }
\end{aligned}
$$

Comparing the coefficient of $i^{n}$, we conclude that

$$
\operatorname{Vol}(\Delta)\left[\frac{1}{2} \int_{\partial \Delta} \mathbf{x} d \sigma+s_{i} \int_{\Delta} \theta_{\Delta} \mathbf{x} d x\right]=\frac{1}{2} \operatorname{Vol}(\partial \Delta) \int_{\Delta} \mathbf{x} d x .
$$

Since $\theta_{\Delta}$ is the potential function of the extremal vector field, it holds

$$
\int_{\partial \Delta} x_{k} d \sigma-\int_{\Delta}\left(\frac{\operatorname{Vol}(\partial \Delta)}{\operatorname{Vol}(\Delta)}+\theta_{\Delta}\right) x_{k} d x=0 \text { for } k=1, \ldots, n .
$$

Hence we have $s_{i}=-\frac{1}{2}+O\left(i^{-1}\right)$.

Now we prove Theorem 1.11 . 
Proof of Theorem 1.11. For any $i \in \mathbb{Z}_{+}$and $g \in P L(\Delta, i)$, by Lemma 3.13 .

$$
\begin{aligned}
\mathcal{Q}_{\Delta}(i, g)= & E_{\Delta}(i) \int_{\Delta} g d x-\operatorname{Vol}(\Delta) \sum_{\mathbf{a} \in \Delta \cap(\mathbb{Z} / i)^{n}}\left(-\frac{\theta_{V}(\mathbf{a})-\bar{\theta}_{i \Delta}}{2 i}+1+O\left(i^{-2}\right)\right) g(\mathbf{a}) \\
= & \left(\operatorname{Vol}(\Delta) i^{n}+\frac{\operatorname{Vol}(\partial \Delta)}{2} i^{n-1}+O\left(i^{n-2}\right)\right) \int_{\Delta} g d x+i^{n-1} \operatorname{Vol}(\Delta) \int_{\Delta} \frac{\theta_{V}-\bar{\theta}_{i \Delta}}{2} g d x \\
& +O\left(i^{n-2}\right)-\left(i^{n} \operatorname{Vol}(\Delta) \int_{\Delta} g d x+\frac{i^{n-1} \operatorname{Vol}(\Delta)}{2} \int_{\partial \Delta} g d \sigma+O\left(i^{n-2}\right)\right)
\end{aligned}
$$

Note that $\bar{\theta}_{i \Delta}=O\left(i^{-1}\right)$, then

$$
\begin{aligned}
\mathcal{Q}_{\Delta}(i, g) & =-\frac{\operatorname{Vol}(\Delta)}{2}\left[\int_{\partial \Delta} g d \sigma-\int_{\Delta}\left(\frac{\operatorname{Vol}(\partial \Delta)}{\operatorname{Vol}(\Delta)}+\theta_{\Delta}\right) g d x\right] i^{n-1}+O\left(i^{n-2}\right) \\
& =-\frac{\operatorname{Vol}(\Delta)}{2} \mathcal{L}_{\Delta}(g) i^{n-1}+O\left(i^{n-2}\right) .
\end{aligned}
$$

By the assumption, $\mathcal{Q}_{\Delta}(i, g) \geq 0$ when $i$ is sufficiently large. This implies $\mathcal{L}_{\Delta}(g) \leq 0$. Hence, $\mathcal{L}_{\Delta}(u) \geq 0$ for any piecewise linear convex function $u$. The theorem is proved.

\section{REDUCTION OF RELATIVE CHOW STABILITY: AN ALTERNATIVE APPROACH}

The asymptotic Chow stability can also be described through the Chow weight of $\mathbb{C}^{*}$ actions induced by test configurations [12, 35]. In this section, we derive an alternative reduction of relative Chow stability of toric manifolds by investigating the normalized weight in [35] for toric degenerations.

First, we recall the notion of a test configuration [12]. A test configuration for a polarized scheme $(X, L)$ is a polarized scheme $(\mathfrak{X}, \mathfrak{L})$ with:

- a $\mathbb{C}^{*}$-action and a proper flat morphism $\pi: \mathfrak{X} \rightarrow \mathbb{C}$ which is $\mathbb{C}^{*}$-equivariant for the usual action on $\mathbb{C}$,

- a $\mathbb{C}^{*}$-equivariant line bundle $\mathfrak{L} \rightarrow \mathfrak{X}$ which is ample over all fibers of $\pi$ such that for $z \neq 0,(X, L)$ is isomorphic to $\left(\mathfrak{X}_{z}, \mathfrak{L}_{z}\right), \mathfrak{L}_{z}=\left.\mathfrak{L}\right|_{\mathfrak{X}_{z}}$.

$(\mathfrak{X}, \mathfrak{L})$ is called product if $\mathfrak{X}=X \times \mathbb{C}$, and trivial if in addition the $\mathbb{C}^{*}$ acts only on $\mathbb{C}$.

It is shown in [35] that, for any $i \in \mathbb{Z}_{+}$, the data of a test configuration for $\left(X, L^{i}\right)$ gives a $\mathbb{C}^{*}$ - action of $G L\left(d_{i}, \mathbb{C}\right)$ and vice versa, where $d_{i}=\operatorname{dim} H^{0}\left(X, L^{i}\right)$. Let $\left(\mathfrak{X}_{0}, \mathfrak{L}_{0}\right)$ be the central fiber. For any $r \in \mathbb{Z}_{+}$, let $k=r i$. Let $w(k)=w(r i)$ be the total weight of the induced $\mathbb{C}^{*}$-action on $H^{0}\left(\mathfrak{X}_{0}, \mathfrak{L}_{0}^{r}\right)$. As in [35], we define the normalized weight $\tilde{w}_{i, k}$ by

$$
\tilde{w}_{i, k}=w(k) i d_{i}-w(i) k d_{k} .
$$

By general algebraic theory, $\tilde{w}_{i, k}$ is a polynomial of degree $n+1$ in $k$, for $k \gg 0$. Write

$$
\tilde{w}_{i, k}=\sum_{j=1}^{n+1} e_{j}(i) k^{j} .
$$

Then the leading term $e_{n+1}(i) i^{n+1}(n+1)$ ! is the Chow weight. Then using the HibertMumford criterion for the $\mathbb{C}^{*}$-actions, Chow stability is described as follows.

Theorem 4.1. ([35]) A polarized variety $(X, L)$ is Chow stable with respect to $i$ if for any nontrivial test configuration for $\left(X, L^{i}\right), e_{n+1}(i)>0 .(X, L)$ is asymptotically Chow 
stable if there exists $i_{0}$ such that for $i \geqslant i_{0}$, any nontrivial test configuration for $\left(X, L^{i}\right)$ has $e_{n+1}(i)>0$.

Now we consider toric manifolds. Recall that a piecewise linear convex function $u=$ $\max \left\{f_{1}, \ldots, f_{\ell}\right\}$ is called rational if $f_{k}=\sum a_{k, i} x_{i}+c_{k}, k=1, \ldots, \ell$, for some vectors $\left(a_{k, 1}, \ldots, a_{k, n}\right) \in \mathbb{R}^{n}$ and some numbers $c_{k} \in \mathbb{R}$ such that all $a_{k, i}$ and $c_{k}$ are rational. According to [12], a toric degeneration for $\left(X_{\Delta}, L_{\Delta}^{i}\right)$ is a test configuration induced by a rational piecewise linear convex function $u$ on $\Delta$, such that $i Q$ is a lattice polytope in $\mathbb{R}^{n+1}=\mathbb{R}^{n} \times \mathbb{R}$. Here $R$ is an integer such that $u \leqslant R$ and

$$
Q=\{(\mathbf{x}, t) \mid \mathbf{x} \in \Delta, 0<t<R-u(\mathbf{x})\} .
$$

Denote the set of all rational piecewise linear convex functions satisfying the above condition by $P L_{i}(\Delta)_{\mathbb{Q}}$.

Lemma 4.2. The Chow weight of the toric degeneration for $\left(X_{\Delta}, L_{\Delta}^{i}\right)$ induced by $u$ is given by $e_{n+1}(i)=-i \mathcal{P}_{\Delta}(i, u)$, where

$$
\mathcal{P}_{\Delta}(i, u)=E_{\Delta}(i) \int_{\Delta} u d x-\operatorname{Vol}(\Delta) \sum_{\mathbf{a} \in \Delta \cap(\mathbb{Z} / i)^{n}} u(\mathbf{a}) .
$$

Proof. By computation [12, 47],

$$
\begin{aligned}
d_{i} & =E_{\Delta}(i), \\
d_{k} & =E_{\Delta}(k)=\operatorname{Vol}(\Delta) k^{n}+\frac{\operatorname{Vol}(\partial \Delta)}{2} k^{n-1}+\cdots+1, \\
w(i) & =i \sum_{\mathbf{a} \in \Delta \cap(\mathbb{Z} / i)^{n}}(R-u)(\mathbf{a}), \\
w(k) & =k^{n+1} \int_{\Delta}(R-u) d x+\frac{k^{n}}{2} \int_{\partial \Delta}(R-u) d \sigma+\cdots .
\end{aligned}
$$

Substituting them into 4.1 , we get

$$
\begin{aligned}
e_{n+1}(i) & =i E_{\Delta}(i) \int_{\Delta}(R-u) d x-\operatorname{Vol}(\Delta) i \sum_{\mathbf{a} \in \Delta \cap(\mathbb{Z} / i)^{n}}(R-u)(\mathbf{a}) \\
& =i\left[E_{\Delta}(i) \int_{\Delta}(-u) d x-\operatorname{Vol}(\Delta) \sum_{\mathbf{a} \in \Delta \cap(\mathbb{Z} / i)^{n}}(-u)(\mathbf{a})\right] .
\end{aligned}
$$

More generally, for the purpose of relative stability, i.e. when $V \neq 0$, we consider the toric degenerations perpendicular to the $\mathbb{C}^{*}$-action $\beta$ induced by $V$. We use the notations of toric data in Section 3. Following [25], we view the Lie algebra of $T_{i \Delta}^{\mathbb{C}}$ as a subalgebra of $\operatorname{sl}\left(E_{\Delta}(i), \mathbb{C}\right)$ by considering the traceless part. The inner product $\langle,\rangle_{i}$ on $\operatorname{sl}\left(E_{\Delta}(i), \mathbb{C}\right)$ is given by

$$
\langle A, B\rangle_{i}=\frac{\operatorname{Tr}(A B)}{i^{n+2}} .
$$

Now let $\alpha$ be the $\mathbb{C}^{*}$-action on the central fiber induced by the toric degeneration. According to [47], the infinitesimal generators of $\alpha$ and $\beta$ are

$$
\operatorname{diag}\left(i(R-u)\left(\mathbf{a}_{1}\right), \cdots, i(R-u)\left(\mathbf{a}_{E_{\Delta}(i)}\right)\right), \operatorname{diag}\left(i \theta_{\Delta}\left(\mathbf{a}_{1}\right), \cdots, i \theta_{\Delta}\left(\mathbf{a}_{E_{\Delta}(i)}\right)\right)
$$


respectively. By considering the traceless parts of them with 4.3 , we call the toric degeneration is perpendicular to $\beta$ if

$$
\sum_{\mathbf{a} \in \Delta \cap(\mathbb{Z} / i)^{n}}(R-u)(\mathbf{a})\left(\theta_{\Delta}(\mathbf{a})-\bar{\theta}_{i \Delta}\right)=\sum_{\mathbf{a} \in \Delta \cap(\mathbb{Z} / i)^{n}} u(\mathbf{a})\left(\theta_{\Delta}(\mathbf{a})-\bar{\theta}_{i \Delta}\right)=0 .
$$

In view of (4.2), we have:

Definition 4.3. $\left(X_{\Delta}, L_{\Delta}\right)$ is called asymptotically relatively Chow semistable for toric degenerations if there exists $i_{0} \in \mathbb{Z}_{+}$, such that when $i \geqslant i_{0}$, the Chow weight of any toric degeneration for $\left(X_{\Delta}, L_{\Delta}^{i}\right)$ is nonnegative. Furthermore, it is called asymptotically relatively Chow stable for toric degenerations if the Chow weight is positive for any nontrivial toric degeneration for $\left(X_{\Delta}, L_{\Delta}^{i}\right)$.

Proposition 4.4. $\left(X_{\Delta}, L_{\Delta}\right)$ is asymptotically relatively Chow semistable for toric degenerations if $(1.5)$ holds and there exists $i_{0} \in \mathbb{Z}_{+}$, such that when $i \geqslant i_{0}$, for any $u \in P L_{i}(\Delta)_{\mathbb{Q}}$ satisfying (4.4), $\mathcal{Q}_{\Delta}(i, u) \leqslant 0$.

Proof. We consider the projection of $u$ onto the perpendicular space. Let

$$
\tilde{u}=u-\frac{\sum_{\mathbf{a} \in \Delta \cap(\mathbb{Z} / i)^{n}} u(\mathbf{a})\left(\theta_{\Delta}(\mathbf{a})-\bar{\theta}_{i \Delta}\right)}{\sum_{\mathbf{a} \in \Delta \cap(\mathbb{Z} / i)^{n}}\left(\theta_{\Delta}(\mathbf{a})-\bar{\theta}_{i \Delta}\right)^{2}}\left(\theta_{\Delta}-\bar{\theta}_{i \Delta}\right) .
$$

Then there exist $r \in \mathbb{Z}_{+}$such that $r \tilde{u}$ induces a toric degeneration for $\left(X_{\Delta}, L_{\Delta}^{i}\right)$ perpendicular to $\beta$. By (4.2), we have

$$
\begin{aligned}
\mathcal{P}_{\Delta}(i, r \tilde{u}) & =E_{\Delta}(i) \int_{\Delta} r \tilde{u} d x-\operatorname{Vol}(\Delta) \sum_{\mathbf{a} \in \Delta \cap(\mathbb{Z} / i)^{n}} r \tilde{u}(\mathbf{a}) \\
& =E_{\Delta}(i) \int_{\Delta} r u d x-\operatorname{Vol}(\Delta) \sum_{\mathbf{a} \in \Delta \cap(\mathbb{Z} / i)^{n}}\left(1+s_{i} \tilde{\theta}_{i \Delta}(\mathbf{a})\right) r u(\mathbf{a})=r \mathcal{Q}_{\Delta}(i, u),
\end{aligned}
$$

where

$$
s_{i}=\frac{i \bar{\theta}_{i \Delta} E_{\Delta}(i)}{\sum_{\mathbf{a} \in \Delta \cap(\mathbb{Z} / i)^{n}}\left(\theta_{\Delta}(\mathbf{a})-\bar{\theta}_{i \Delta}\right)^{2}} .
$$

In the above deduction, we used the normalization condition $\int_{\Delta} \theta_{\Delta} d x=0$. The necessary condition (1.5) for asymptotic relative Chow semistability in the toric sense in the last section can also be recovered by substituting $u=x_{j}$ and $-x_{j}$ into the above for $j=1, \ldots, n$. It is also easy to see that in (1.5), if the $s_{i}$ exists, it can be given by (4.5).

Remark 4.5. The sign differs from (1.8) because we consider convex functions here, while in (1.8) $g$ is a concave function.

\section{EXAMPLES}

Finally we provide many interesting examples which will support our understanding of stabilities. Here we will mainly concentrate on toric Fano manifolds. 
5.1. Relative $K$-stability of toric Fano threefolds. In this section, we shall determine the potential $\theta_{\Delta}$ of the extremal vector field $V$ of toric Fano threefolds in symplectic coordinates and verify the relative $K$-stability or instability by Theorem 1.4 .

If $V$ is given by $V=\sum_{i=1}^{n} a_{i} \frac{\partial}{\partial w_{i}}$ in the affine logarithm coordinates $\left(w_{1}, \ldots, w_{n}\right)$, then the potential function $\theta_{\Delta}$ is given by $\theta_{\Delta}=\sum_{i=1}^{n} a_{i} x_{i}+c$ for some constant $c$. There are several ways to compute $\theta_{\Delta}$. The most general one is to use the linear functional (1.2). In order to determine constants $a_{i}$ and $c$, one can solve the $n+1$-linear system

$$
\mathcal{L}_{\Delta}(1)=0, \mathcal{L}_{\Delta}\left(x_{i}\right)=0 \quad \text { for } \quad i=1, \ldots, n .
$$

In Fano case, we have a more efficient algorithm. By [2], $\varphi$ in (2.4) can be given by

$$
\varphi=\log \left(\sum_{i=1}^{m} e^{\left\langle p^{(i)}, y\right\rangle}\right)
$$

where $p^{(1)}, \ldots, p^{(m)}$ are the vertices of $\Delta$. The Fano assumption implies

$$
\left|\varphi+\log \operatorname{det}\left(\varphi_{i j}\right)\right|<\infty .
$$

We recall another normalization on the potentials of holomorphic vector fields. Let $v$ be a holomorphic vector field on $X$ and $\theta_{v}^{\prime}\left(\omega_{g}\right)$ be the potential function determined by

$$
i_{v} \omega_{g}=\sqrt{-1} \bar{\partial} \theta_{v}^{\prime}\left(\omega_{g}\right), \quad \text { and } \quad \int_{X} \theta_{v}^{\prime}\left(\omega_{g}\right) e^{h_{g}} \frac{\omega_{g}^{n}}{n !}=0
$$

where $h_{g}$ is a Ricci potential of $\omega_{g}$. According to [41], the above $\theta_{v}^{\prime}$ satisfies

$$
\theta_{v}^{\prime}=-\Delta_{g} \theta_{v}^{\prime}-v\left(h_{g}\right) \text {, }
$$

where $\Delta_{g}$ is the Laplacian. Assume $v=\sum_{i=1}^{n} a_{i} z_{i} \frac{\partial}{\partial z_{i}}=\sum_{i=1}^{n} a_{i} \frac{\partial}{\partial w_{i}}$ on the torus orbit, where $a_{i} \in \mathbb{R}$. Then we have $\theta_{v}^{\prime}\left(\omega_{g}\right)=\sum_{i=1}^{n} a_{i} x_{i}$ in the symplectic coordinates by a simple observation from (5.2). In particular, $\theta_{\frac{\partial}{\partial w_{i}}}^{\prime}\left(\omega_{g}\right)=x_{i}$ for $i=1, \ldots, n$. This simple fact has been used in [47]. Then by (5.5), we can compute the Futaki invariant by

$$
F(v)=\int_{X} v\left(h_{g}\right) \omega^{n}=-\int_{X} \theta_{v}^{\prime} \omega^{n}=-(2 \pi)^{2} \int_{\Delta} \theta_{v}^{\prime} d x .
$$

The first step of the algorithm is to determine $a_{1}, \ldots, a_{n}$ by $(5.6)$ and Lemma 2.1. Then

$$
c=-\frac{1}{\operatorname{Vol}(\Delta)} \int_{\Delta} \sum_{i} a_{i} x_{i} d x
$$

by the normalization condition. An alternative method to compute $a_{1}, \ldots, a_{n}$ was also given by Nakagawa. He gave a combinatorial formula for Futaki invariant and the generalized Killing form of toric Fano orbifolds in [27]. As an application, he computed the extremal vector field in the anti-canonical class on a toric Fano manifold $X$ with $\operatorname{dim} X \leqslant 4$. In order to prove Theorem 1.5, we shall use his result on toric Fano threefolds directly.

The main goal of this section is to prove the following proposition. 
Proposition 5.1. Let $X$ be a toric Fano threefold with anti-canonical polarization.

(a) If $X=\mathcal{B}_{2}$, then $X$ is relatively (strongly) $K$-stable in the toric sense in the anticanonical class.

(b) If $X=\mathcal{B}_{1}$, then $X$ is relatively $K$-unstable in its first Chern class.

Once Proposition 5.1 has been proved, other cases are similar and further details are left to the reader ${ }^{1}$. In Table 1 and Table 2 we give the list of all results proved in Theorem 1.5 .

Proof. (a) Let $e_{i}(i=1,2,3)$ be the standard basis of $\mathfrak{N} \cong \mathbb{Z}^{3}$. Let $\Sigma$ be the complete fan in $\mathfrak{N}_{\mathbb{R}} \cong \mathbb{R}^{3}$ whose 1-dimensional cones are given by $\Sigma(1)=\left\{\sigma_{1}, \sigma_{2}, \sigma_{3}, \sigma_{4}, \sigma_{5}\right\}$ where

$$
\begin{aligned}
& \sigma_{1}=\operatorname{Cone}\left(e_{1}\right), \quad \sigma_{2}=\operatorname{Cone}\left(e_{2}\right), \quad \sigma_{3}=\operatorname{Cone}\left(e_{3}\right), \\
& \sigma_{4}=\operatorname{Cone}\left(-e_{3}\right), \quad \text { and } \quad \sigma_{5}=\operatorname{Cone}\left(-e_{1}-e_{2}-e_{3}\right) .
\end{aligned}
$$

Then the associated toric manifold $X$ is $\mathbb{C} P\left(\mathcal{O}_{\mathbb{C} P^{2}} \oplus \mathcal{O}_{\mathbb{C} P^{2}}(1)\right)$ and

$$
\Delta=\left\{\left(x_{1}, x_{2}, x_{3}\right) \in \mathbb{R}^{3} \mid x_{1} \geqslant-1, x_{2} \geqslant-1, x_{3} \geqslant-1, x_{3} \leqslant 1, x_{1}+x_{2}+x_{3} \leqslant 1\right\} .
$$

Hence $\operatorname{Vol}(\Delta)=\frac{28}{3}$. Let $V \in \eta_{c}(X)$ be the extremal vector field in the anti-canonical class and $\theta_{\Delta}$ be the potential function of $V$. Then $\theta_{\Delta}=-\frac{70}{97} x_{3}+c$ for some constant $c$. (See [27], Section 6, Table 2). Since we have $\int_{\Delta} x_{3}=-2$, we conclude that $c=-\frac{15}{97}$ by (5.7). Thus $\theta_{\Delta}=-\frac{70}{97} x_{3}-\frac{15}{97}$ and

$$
\Delta^{-}=\left\{\left(x_{1}, x_{2}, x_{3}\right) \in \Delta \mid-\frac{112}{97}-\frac{70}{97} x_{3} \geqslant 0\right\}=\emptyset .
$$

$X$ satisfies the condition (1.3). The assertion is verified.

(b) Let $\Sigma$ be the complete fan in $N_{\mathbb{R}} \cong \mathbb{R}^{3}$ whose 1-dimensional cones are given by $\Sigma(1)=$ $\left\{\sigma_{1}, \ldots, \sigma_{5}\right\}$ where

$$
\begin{aligned}
& \sigma_{1}=\operatorname{Cone}\left(e_{1}\right), \quad \sigma_{2}=\operatorname{Cone}\left(e_{2}\right), \quad \sigma_{3}=\operatorname{Cone}\left(e_{3}\right), \\
& \sigma_{4}=\operatorname{Cone}\left(-e_{3}\right), \quad \text { and } \quad \sigma_{5}=\operatorname{Cone}\left(-e_{1}-e_{2}-2 e_{3}\right) .
\end{aligned}
$$

Then we readily see that $X=\mathbb{C} P\left(\mathcal{O}_{\mathbb{C} P^{2}} \oplus \mathcal{O}_{\mathbb{C} P^{2}}(2)\right)$ and $\Delta=\operatorname{Conv}\left\{e_{3}^{*}-e_{2}^{*}, 4 e_{1}^{*}-e_{2}^{*}-e_{3}^{*},-e_{1}^{*}-e_{2}^{*}-e_{3}^{*}, e_{3}^{*}-e_{1}^{*}-e_{2}^{*}, 4 e_{2}^{*}-e_{1}^{*}-e_{3}^{*}, e_{3}^{*}-e_{1}^{*}\right\}$, where $e_{i}^{*}$ is the dual basis of $e_{i}$. Then $\operatorname{Vol}(\Delta)=\frac{31}{3}$ and $\theta_{\Delta}=-\frac{620}{349} x_{3}+c$ for some constant c. Since $\int_{\Delta} x_{3}=-4$, we obtain $c=-\frac{240}{349}$. Hence we conclude $\theta_{\Delta}=-\frac{620}{349} x_{3}-\frac{240}{349}$ and

$$
\begin{aligned}
\Delta^{-} & =\left\{\left(x_{1}, x_{2}, x_{3}\right) \in \Delta \mid-\frac{589}{349}-\frac{620}{349} x_{3} \geqslant 0\right\} \\
& =\operatorname{Conv}\left\{\begin{array}{ccc}
4 e_{1}^{*}-e_{2}^{*}-e_{3}^{*}, & \frac{39}{10} e_{1}^{*}-e_{2}^{*}-\frac{19}{20} e_{3}^{*}, & -e_{1}^{*}-e_{2}^{*}-\frac{19}{20} e_{3}^{*} \\
-e_{1}^{*}+\frac{39}{10} e_{2}^{*}-\frac{19}{20} e_{3}^{*}, & -e_{1}^{*}-e_{2}^{*}-e_{3}^{*}, & -e_{1}^{*}+4 e_{2}^{*}-e_{3}^{*}
\end{array}\right\} .
\end{aligned}
$$

Thus

$$
\operatorname{Vol}\left(\Delta^{-}\right)=\frac{7351}{12000}
$$

\footnotetext{
${ }^{1}$ In the practical computation we used packages (i) Normaliz and (ii) Polymake. These packages are available at [4] and [20] respectively.
} 
Now we shall verify Condition (1.4). First we note that $\int_{\Delta^{-}} x_{3} d x=-\frac{96197}{4}$ and $\int_{\Delta^{-}} x_{3}^{2} d x=\frac{1828273}{5}$. Hence one can see that

$$
\int_{\Delta^{-}}\left(1-\theta_{\Delta}\right)^{2} d x=\int_{\Delta^{-}}\left(\frac{589}{349}+\frac{620}{349} x_{3}\right)^{2} d x=\frac{1475918766336271}{1461612000} .
$$

Plugging this and (5.8) into (1.4), we obtain the desired result because $1-c=\frac{589}{349}$.

Remark 5.2. It is also interesting to ask if the method in Section 5.1 can determine all toric Fano fourfolds. However, we have some technical difficulties on computing (1.4). That is, the vertices of $\Delta^{-}$can be very complicated which leads to the overflow of the software Normaliz when we compute the term $\int_{\Delta^{-}}\left(1-\theta_{\Delta}\right)^{2} d x$ while some of them are still computable. Our practical computation shows that the stability of all computable ones can be determined by Theorem 1.4. That is among all toric Fano fourfolds (124 deformation classes), there are 52 types of relative $K$-stable ones, 15 types of relative $K$-unstable ones, and 57 types of undistinguishable ones. We expect that the 57 types of undistinguishable ones can be settled by using more advanced software or improvement of the criterion in Thereom 1.4 .

5.2. Relative Chow stability. We study relatively Chow unstable examples of toric Fano manifolds. First, we recall the example found by Nill and Paffenholz [29] which is isomorphic to $\mathbb{P}\left(\mathcal{O}_{W} \oplus \mathcal{O}_{W}(-1,-1,-1,2)\right)=: X_{\mathrm{NP}}$ where $W=\left(\mathbb{C} P^{1}\right)^{3} \times \mathbb{C} P^{3} . X_{\mathrm{NP}}$ is a non-symmetric Kähler-Einstein toric Fano 7-fold. In [34], Ono, Sano and Yotsutani showed that $\left(X_{\mathrm{NP}},-K_{X_{\mathrm{NP}}}\right)$ is asymptotically Chow unstable. Later Yotsutani observed that $X_{\mathrm{NP}}$ is Chow unstable w.r.t. $-K_{X_{\mathrm{NP}}}$ (i.e., $i=1$ ) using (3.8). Meanwhile, Nill and Paffenholz [29] also proved that all toric Kähler-Einstein Fano manifolds are symmetric if $\operatorname{dim} X \leqslant 6$. It is known that if $X$ is a symmetric toric Fano manifold, then $\left(X,-K_{X}\right)$ is asymptotically Chow stable. Hence, the lowest dimension for an anti-canonically polarized Kähler-Einstein toric Fano manifold $\left(X,-K_{X}\right)$ to be (asymptotically) Chow unstable is 7 . However, in the case where $X$ is a Fano orbifold, such an example appears in $\operatorname{dim} X=3$.

Proposition 5.3. There is a Kähler-Einstein toric Fano orbifold $X$ with $\operatorname{dim} X=3$ which is Chow unstable w.r.t. $\left(-K_{X}\right)^{2}$.

Our strategy is the following. For any toric Fano orbifold $X$ with the associated simplicial Fano polytope $\Delta^{\circ} \subseteq \mathfrak{N}_{\mathbb{R}}, X$ admits Kähler-Einstein metric if and only if the Futaki invariant of $X$ vanishes by Shi-Zhu's result [37]. Let $\mathcal{W}(X)$ be the Weyl group of Aut $(X)$ with respect to the maximal torus and $\mathfrak{N}_{\mathbb{R}}^{\mathcal{W}(X)}$ be the $\mathcal{W}(X)$-invariant subspace of $\mathfrak{N}_{\mathbb{R}}$. It is known that $\sum_{v \in \mathcal{V}\left(\Delta^{\circ}\right)} v \in \mathfrak{N}_{\mathbb{R}}^{\mathcal{W}(X)}$ (see [28, Chapter 5]). Here $\mathcal{V}\left(\Delta^{\circ}\right)$ denotes the set of vertices of $\Delta^{\circ}$. Note that $X$ is symmetric if and only if $\mathfrak{N}_{\mathbb{R}}^{\mathcal{W}(X)}=\{0\}$. Hence we may consider toric Fano orbifolds with the vanishing Futaki invariant satisfying $\sum_{v \in \mathcal{V}\left(\Delta^{\circ}\right)} v \neq 0$. Among all 12, 190 3-dimensional toric Fano orbifolds (i.e., $\mathbb{Q}$-factorial toric Fano varieties), there are 42 toric Fano orbifolds with the vanishing Futaki invariant. Of these 42 toric Fano orbifolds, there is the only one example satisfying the above conditions. Then it suffices to check (3.8) for the dual moment polytope $\Delta \subseteq \mathfrak{M}_{\mathbb{R}}$ of this one. Note that the Gorenstein index $j_{X}$ is given by minimal $k$ such that $k \Delta$ is a lattice polytope for a fixed canonical Fano polytope $\Delta^{\circ} \subseteq \mathfrak{N}_{\mathbb{R}}[28$, Proposition 2.3.2]. 
Proof of Proposition 5.3 Again we use the same notations as in the proof of Proposition 5.1. We consider the 3-dimensional canonical Fano polytope $\mathrm{e}^{2}$

$\Delta^{\circ}:=\operatorname{Conv}\left\{e_{1}-e_{2}-2 e_{3}, e_{2}+3 e_{3}, e_{1}+e_{2}+3 e_{3}, e_{1}+2 e_{2}+4 e_{3}, e_{2},-2 e_{1}-2 e_{2}-3 e_{3}\right\}$.

The vertices of the dual polytope $\Delta$ are

$-\frac{1}{2} e_{1}^{*}+\frac{5}{2} e_{2}^{*}-e_{3}^{*}, e_{1}^{*}-e_{2}^{*}, 2 e_{2}^{*}-e_{3}^{*}, \frac{1}{2} e_{2}^{*}-\frac{1}{2} e_{3}^{*},-e_{1}^{*},-e_{1}^{*}-e_{2}^{*}+\frac{1}{2} e_{3}^{*}, \frac{3}{2} e_{1}^{*}-e_{2}^{*},-e_{2}^{*}+e_{3}^{*}$.

Thus $\Delta$ is a simple polytope and $j_{X_{\Delta}}=2$. Setting $\widetilde{\Delta}:=2 \Delta$, we compute the Chow weight of $\widetilde{\Delta}$. We readily see that

$E_{\widetilde{\Delta}}(i)=12 i^{3}+9 i^{2}+3 i+1, \quad \operatorname{Vol}(\widetilde{\Delta})=12, \quad \int_{\widetilde{\Delta}} \mathbf{x} d x=(0,0,0), \quad \int_{\partial \widetilde{\Delta}} \mathbf{x} d \sigma=(0,0,0)$

and

$$
\sum_{\mathbf{a} \in \widetilde{\Delta} \cap \mathbb{Z}^{3}} \mathbf{a}=(0,1,-1), \quad \sum_{\mathbf{a} \in 2 \widetilde{\Delta} \cap \mathbb{Z}^{3}} \mathbf{a}=(0,3,-3), \quad \sum_{\mathbf{a} \in 3 \widetilde{\Delta} \cap \mathbb{Z}^{3}} \mathbf{a}=(0,6,-6)
$$

hold. This implies that

$$
\int_{\partial \widetilde{\Delta}} \mathbf{x} d \sigma-\frac{\operatorname{Vol}(\partial \widetilde{\Delta})}{\operatorname{Vol}(\widetilde{\Delta})} \int_{\widetilde{\Delta}} \mathbf{x} d x=(0,0,0)
$$

i.e. the Futaki invariant vanishes. However obviously,

$$
\sum_{\mathbf{a} \in \widetilde{\Delta} \cap \mathbb{Z}^{3}} \mathbf{a} \neq \frac{E_{\widetilde{\Delta}}(1)}{\operatorname{Vol}(\widetilde{\Delta})} \int_{\widetilde{\Delta}} \mathbf{x} d x
$$

By (3.8), the 2-Gorenstein toric Fano variety $\left(X_{\Delta},\left(-K_{X_{\Delta}}\right)^{2}\right)$ is Chow unstable.

In [3], Berman proved that a $\mathbb{Q}$-Fano variety $X$ admitting Kähler-Einstein metrics is $K$-stable. Hence Remark 3.12 (2) gives the following.

Corollary 5.4. The example in Proposition 5.3 is $K$-stable but asymptotically Chow unstable.

Next, we consider the general case with the nontrivial Futaki invariant. We see that a smooth counter-example appears in $\operatorname{dim} X=3$.

Proposition 5.5. Let $X$ be a toric Fano threefold which is isomorphic to $\mathcal{E}_{4}$. Then $\left(X,-K_{X}\right)$ is relatively $K$-stable but it is asymptotically relatively Chow unstable.

Proof. It suffices to see that $(1.5)$ is not satisfied. The corresponding 3-dimensional moment polytope is listed in Table 1 . Thus we have

$$
E_{\Delta}(i)=\frac{20}{3} i^{3}+10 i^{2}+\frac{16}{3} i+1 \quad \text { and } \quad \int_{\Delta} \mathbf{x} d x=\left(-\frac{7}{8}, \frac{5}{12}, \frac{5}{24}\right) .
$$

In particular,

$$
\sum_{\mathbf{a} \in \Delta \cap \mathbb{Z}^{3}} \mathbf{a}=(-4,2,1) \quad \text { and } \quad s_{1} \sum_{\mathbf{a} \in \Delta \cap \mathbb{Z}^{3}}\left(\theta_{\Delta}(\mathbf{a})-\bar{\theta}_{\Delta}\right) \mathbf{a}=s_{1}\left(-\frac{11134272}{1816885}, \frac{1079424}{363377}, \frac{539712}{363377}\right)
$$

holds. Thus there is no $s_{1}$ satisfying (1.5). The assertion follows from Corollary 3.11 .

\footnotetext{
${ }^{2}$ ID number in the database [49] is 530571.
} 
TABLE 2. $\theta_{\Delta}$ and $\Delta$ on toric Fano threefolds

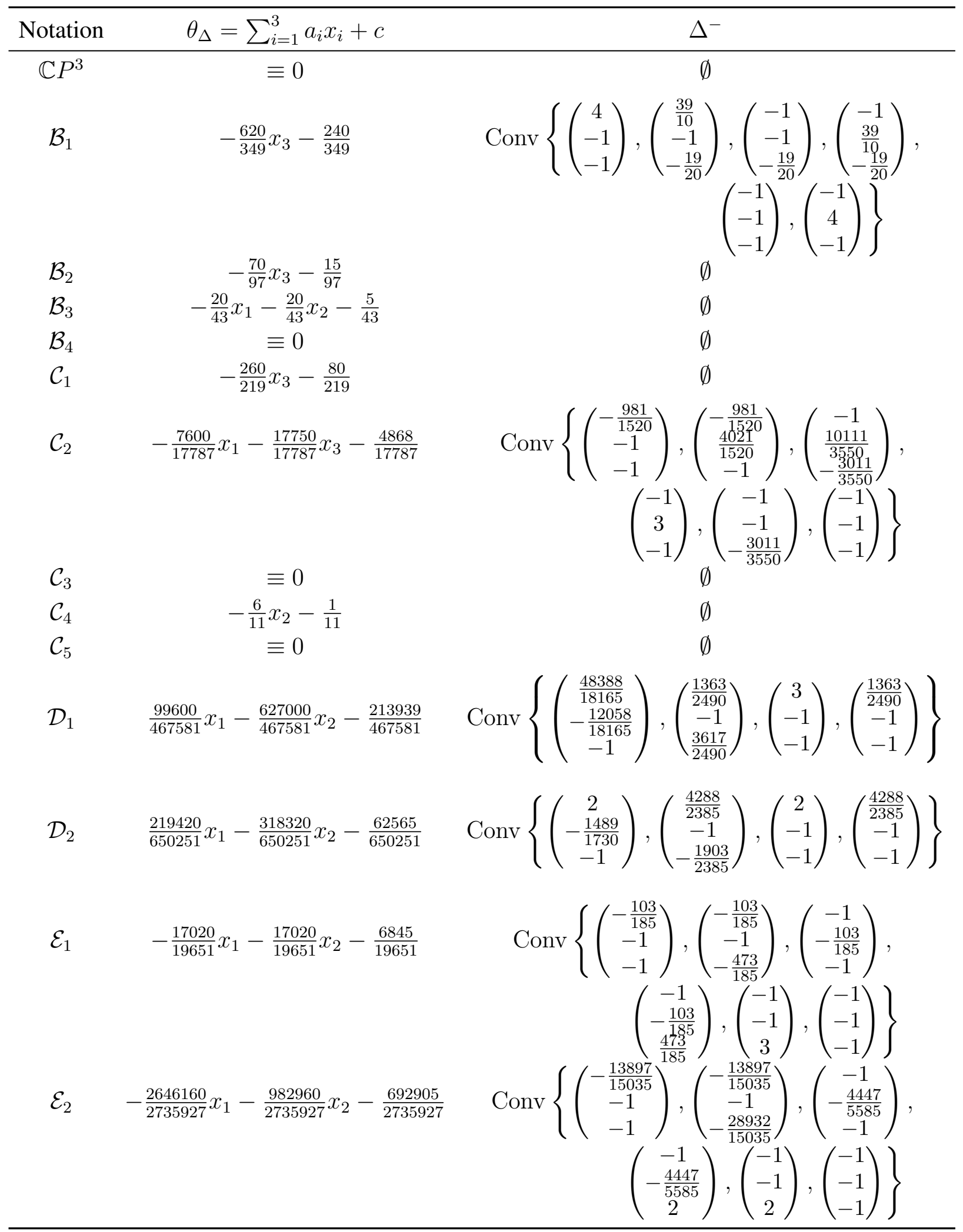




\begin{tabular}{ccc}
\hline Notation & $\theta_{\Delta}$ & $\Delta^{-}$ \\
\hline $\mathcal{E}_{3}$ & $-\frac{168}{409} x_{1}-\frac{168}{409} x_{2}-\frac{32}{409}$ & $\emptyset$ \\
$\mathcal{E}_{4}$ & $-\frac{34208}{78995} x_{1}+\frac{7936}{78995} x_{2}-\frac{24929}{394975}$ & $\emptyset$ \\
$\mathcal{F}_{1}$ & $\equiv 0$ & $\emptyset$ \\
$\mathcal{F}_{2}$ & $\frac{36}{67} x_{2}-\frac{5}{67}$ & $\emptyset$ \\
\hline
\end{tabular}

\section{REFERENCES}

[1] V. Batyrev, Troidal Fano 3-folds. Math. USSR-Izv. 19 (1982), 13-25. Izv. Akad. Nauk SSSR 45 (1981), 704-717.

[2] V. Batyrev and E. Selivanova, Einstein-Kähler metrics on symmetric toric Fano manifolds. J. Reine Angew. Math. 512 (1999), 225-236.

[3] R. Berman, K-stability of Q-Fano varieties admitting Kahler-Einstein metrics. Invent. Math. 203 (2016), 973-1025.

[4] W. Bruns, B. Ichim, T. Römer and C. Söger, Normaliz. Algorithms for rational cones and affine monoids. Available at http://www. math.uos.de/normaliz.

[5] E. Calabi, Extremal Kähler metrics, Seminar on differential geometry, Ann. of Math Studies, Princeton Univ. Press, 102 (1982), 259-290.

[6] B.H. Chen, A.M. Li and L. Sheng, Extremal metrics on toric surfaces. arXiv:1008.2607.

[7] X. X. Chen, C. LeBrun and B. Weber, On conformally Kähler-Einstein manifolds. J. Amer. Math. Soc. 21 (2008), 1137-1168.

[8] D. A. Cox, J. B. Little and H. K. Schenck. Toric varieties. Graduate Studies in Mathematics, 124. American Mathematical Society, Providence, RI, 2011. xxiv+841 pp.

[9] I. Dolgachev, Lectures on invariant theory. London Mathematical Society Lecture Note Series, 296. Cambridge University Press, Cambridge, 2003.

[10] O. Debarre, Fano varieties, Higher dimensional varieties and rational points. Budapest, 2001, Bolyai Society Mathematical Studies 12, 93-132. Springer, Berlin 2003.

[11] S.K. Donaldson, Scalar curvature and projective embeddings, I. J. Diff. Geom. 59 (2001), 479-522.

[12] S.K. Donaldson, Scalar curvature and stability of toric varieties. J. Diff. Geom. 62 (2002), 289-349.

[13] S.K. Donaldson, Extremal metrics on toric surfaces: a continuity method. J. Diff. Geom. 79 (2008), 389-432.

[14] S.K. Donaldson, Constant scalar curvature metrics on toric surfaces. Geom. Funct. Anal. 19 (2009), 83-136.

[15] A. Futaki, Asymptotic Chow semi-stability and integral invariants. Int. J. Math. 15 (2004), 967-979.

[16] A. Futaki, Asymptotic Chow stability in Kähler geometry. Fifth International Congress of Chinese Mathematicians. Part 1, 2, 139-153, AMS/IP Stud. Adv. Math., 51, pt. 1, 2, Amer. Math. Soc., Providence, RI, 2012.

[17] A. Futaki and T. Mabuchi, Bilinear forms and extremal Kähler vector fields associated with Kähler class. Math. Ann. 301 (1995), 199-210.

[18] A. Futaki, H. Ono and Y. Sano, Hilbert series and obstructions to asymptotic semistability. Adv. Math. 226 (2011), 254-284.

[19] I. M. Gelfand, M. M. Kapranov, and A. V. Zelevinsky, Discriminants, resultants, and multidimensional determinants. Mathematics: Theory \& Applications. Birkhäuser Boston Inc., Boston, MA, 1994.

[20] E. Gawrilow and M. Joswig, Polymake Version 2.9.8-Convex polytopes, polyhedra, simplicial complexes, matroids, fans, and tropical objects, Available at wwwopt.mathematik.tudarmstadt.de/polymake/doku.php, 1997-present.

[21] M. M. Kapranov, B. Sturmfels and A. V. Zelevinsky, Chow polytopes and general resultants. Duke Math. J. 67 (1992), 189-218.

[22] A. M. Kasprzyk, Canonical toric Fano threefolds. Canad. J. Math. 62 (2010), 1293-1309. 
[23] T. Mabuchi, Stability of extremal Kähler manifolds. Osaka J. Math. 41 (2004), 563-582.

[24] T. Mabuchi, An energy-theoretic approach to the Hitchin-Kobayashi correspondence for manifolds. I. Invent. Math. 159 (2005), 225-243.

[25] T. Mabuchi, Relative stability and extremal metrics. J. Math. Soc. Japan 66 (2014), 535-563.

[26] T. Mabuchi, Asymptotic polybalanced kernels on extremal Kaehler manifolds. arXiv:1610.09632.

[27] Y. Nakagawa, Combinatorial formulae for Futaki characters and generalized killing forms of toric Fano orbifolds. The Third Pacific Rim Geometry Conference (Seoul, 1996), 223-260, Monogr. Geom. Topology, 25, Int. Press, Cambridge, MA, 1998.

[28] B. Nill, Gorenstein toric Fano varieties. Dissertation, Universität Tübingen, 2005. Available at http://tobias-lib.uni-tuebingen.de/volltexte/2005/1888

[29] B. Nill and A. Paffenholz, Examples of Kähler-Eisntein toric Fano manifolds associated to nonsymmetric reflexive polytopes. Beitr. Algebra. Geom 52 (2011), 297-304.

[30] M. Obro, An algorithm for the classification of smooth Fano polytopes. arXiv:0704.0049 (2007).

[31] Y. Odaka, The Calabi conjecture and K-stability. Int. Math. Res. Not. IMRN 2012, 2272-2288.

[32] H. Ono, A necessary condition for Chow semistability of polarized toric manifolds. J. Math. Soc. Japan. 63 (2011), 1377-1389.

[33] H. Ono, Algebro-geometric semistability of polarized toric manifolds. Asian J. Math. 17 (2013), 609616.

[34] H. Ono, Y. Sano and N. Yotsutani, An example of an asymptotically Chow unstable manifold with constant scalar curvature. Ann. Inst. Fourier (Grenoble) 62 (2012), 1265-1287.

[35] J. Ross and R. Thomas, A study of the Hilbert-Mumford criterion for the stability of projective varieties. J. Alg. Geom. 16 (2007), 201-255.

[36] R. Seyyedali, Relative Chow stability and extremal metrics. arXiv:1610.07555.

[37] Y. Shi and X. Zhu, Kähler-Ricci solitons on toric Fano orbifolds. Math. Zeit. 271 (2012), 1241-1251.

[38] G. Székelyhidi, Extremal metrics and K-stability. Dissertation, Imperial college, London, (2006), arXiv:0611002.

[39] G. Székelyhidi, Extremal metrics and K-stability. Bull. London Math. Soc. 39 (2007), 76-84.

[40] G. Tian, Kähler-Einstein metrics with positive scalar curvature. Invent. Math. 130 (1997), 1-39.

[41] G. Tian and X.H. Zhu, A new holomorphic invariant and uniqueness of Kahler-Ricci solitons. Comm. Math. Helv. 77 (2002), 297-325.

[42] A.D. Vedova and F. Zuddas, Scalar curvature and asymptotic Chow stability of projective bundles and blowups. Trans. Amer. Math. Soc. 364 (2012), 6495-6511.

[43] K. Watanabe and M. Watanabe, The classification of Fano 3-folds with torus embeddings. Tokyo J. Math. 5 (1982), 37-48.

[44] X. J. Wang and B. Zhou, Existence and nonexistence of extremal metrics on toric Kähler manifolds. Adv. Math. 226 (2011), 4429-4455.

[45] X. J. Wang and X. Zhu, Kähler-Ricci solitons on toric manifolds with positive first Chern class. Adv. Math. 188 (2004), 87-103.

[46] N. Yotsutani, Facets of secondary polytopes and Chow stability of toric varieties. Osaka J. Math 53, (2016), 751-765.

[47] B. Zhou and X.H. Zhu, Relative $K$-stability and modified K-energy on toric manifolds. Adv. Math. 219 (2008), 1327-1362.

[48] B. Zhou and X.H. Zhu, K-stability on toric manifolds. Proc. Amer. Math. Soc. 136 (2008), 3301-3307.

[49] Graded Ring Database, http://grdb.lboro.ac.uk/forms/toricsmooth.

School of Mathematical Sciences at Fudan University, Shanghai, 200433, P. R. China

E-mail address: naoto-yot sutani@fudan.edu.cn

School of Mathematical Sciences, Peking University, Beijing, 100871, P. R. China; And Mathematical Sciences Institute, The Australian National University, CanberRa, ACT 2601, Australia.

E-mail address: bzhou@pku.edu.cn; bin.zhou@anu.edu.au 